\title{
Resolving the ultrafast dynamics of the anionic green fluorescent protein chromophore in water
}

\author{
Chey M. Jones, ${ }^{1,2}$ Nanna H. List, ${ }^{1,2}$ and Todd J. Martínez ${ }^{1,2, *}$ \\ ${ }^{1}$ Department of Chemistry and The PULSE Institute, Stanford University, Stanford, CA 94305 \\ ${ }^{2}$ SLAC National Accelerator Laboratory, 2575 Sand Hill Road, Menlo Park, CA 94025
}

\begin{abstract}
:
The chromophore of the green fluorescent protein (GFP) is critical for probing environmental influences on fluorescent protein behavior. Using the aqueous system as a bridge between the unconfined vacuum system and a constricting protein scaffold, we investigate the steric and electronic effects of the environment on the photodynamical behavior of the chromophore. Specifically, we perform ab initio multiple spawning to simulate five picoseconds of nonadiabatic dynamics after photoexcitation, resolving the excited-state pathways responsible for internal conversion in the aqueous chromophore. We identify an ultrafast pathway that proceeds through a short-lived (sub-picosecond) imidazolinonetwisted (I-twisted) species and a slower (several picoseconds) channel that proceeds through a longlived phenolate-twisted (P-twisted) intermediate. The molecule navigates the non-equilibrium energy landscape via an aborted hula-twist-like motion towards the one-bond-flip dominated conical intersection seams, as opposed to following the pure one-bond-flip paths proposed by the excited-state equilibrium picture. We interpret our simulations in the context of time-resolved fluorescence experiments, which use short and long time components to describe the fluorescence decay of the aqueous GFP chromophore. Our results suggest that the longer time component is caused by an energetically uphill approach to the P-twisted intersection seam rather than an excited-state barrier to reach the twisted intramolecular charge transfer species. Irrespective of the location of the non-adiabatic population events, the twisted intersection seams are inefficient at facilitating isomerization in aqueous solution. The disordered and homogeneous nature of the aqueous solvent environment facilitates nonselective stabilization with respect to I- and P-twisted species, offering an important foundation for understanding the consequences of selective stabilization in heterogeneous and rigid protein environments.
\end{abstract}




\section{Introduction}

Since their initial characterization in Aequorea victoria, ${ }^{1-5}$ fluorescent proteins (FPs) have emerged as powerful probes for in vivo biological function. ${ }^{6-11}$ The green fluorescent protein ${ }^{8}$ (GFP) is the monarch of fluorescent proteins, and its utility and versatility keep the green fluorophore at the forefront of science. Seminal experiments on the color and protonation state tuning of GFP ${ }^{12-17}$ inspired novel FP biomarkers, with reversible photoswitchable fluorescent proteins propelling the resolution of FPs beyond the diffraction limit. ${ }^{16-18}$ Located at the heart of GFP, a tunable chromophore is responsible for GFP's light emission. Because the native characteristics of GFP can be altered significantly by applying point mutations near the site of the chromophore, ${ }^{8}$ we need a detailed understanding of the chromophore's local steric and electronic environment to optimize the versatility of GFP. Frequently, $p$ hydroxybenzylidene-2,3-dimethylimidazolinone (HBDI), first synthesized by Kojima et al., ${ }^{19}$ serves as a model GFP chromophore. In particular, the anionic form of HBDI (HBDI-) mimics the protonation state of the chromophore responsible for fluorescence in the protein. ${ }^{20-21}$

The overwhelming number of variables in a complex protein environment creates a fundamental roadblock to the rational design of FPs. Due to the rigidity of its 11 -stranded $\beta$-barrel, ${ }^{22-23}$ GFP envelops its chromophore in a highly ordered pocket of amino acids. Stiff protein backbones foster heterogeneous electronic and steric fingerprints that can significantly impact the chromophore's behavior. ${ }^{8}$ Because amino acids in GFP form complex networks of covalent and non-covalent interactions, it is difficult to predict the net effect of various residues acting on the chromophore. To filter out the effects that dictate chromophore behavior, we use HBDI- to monitor the molecule's response to incremental perturbations of the environment. Using the gas-phase dynamics of $\mathrm{HBDI}^{-}$as a baseline, ${ }^{24}$ we identify the influence of specific effects on chromophore behavior. Here, we focus on a key system that unravels the role of environmental influences on GFP chromophore photodynamics - the homogeneous and disordered case of aqueous $\mathrm{HBDI}^{-}$.

Solvated HBDI- straddles the line between the freedom permitted by the gas-phase and the confinement imposed by a protein. In water, $\mathrm{HBDI}^{-}$experiences steric and electronic effects from the solvent, which are absent in vacuo, while in an environment that is less constricting than a protein scaffold. Time-resolved spectroscopies reveal that GFP has an excited-state lifetime on the order of nanoseconds ${ }^{20,25-26}$ and a fluorescence quantum yield near unity. ${ }^{8}$ Yet, HBDI- experiences ultrafast internal conversion in vacuum and in solution at ambient temperature. ${ }^{27-31}$ Twisting around the bridge bonds connecting the imidazolinone (I) and phenolate (P) moieties of the isolated chromophore leads to 
ultrafast deactivation that decreases fluorescence quantum yield. ${ }^{31-33}$ As highlighted in previous computational studies, ${ }^{34-35}$ non-methylated $\mathrm{HBDI}^{-}\left(\mathrm{pHBI}^{-}\right)$undergoes twisted intramolecular charge transfer (TICT) as rotations of the I- and P-moieties become sufficiently large. The direction of charge transfer $(\mathrm{CT})$ on the excited state depends on whether the I-moiety or the P-moiety rotates (about the $\phi_{I} / \phi_{P}$ dihedrals indicated in Figure 1, respectively). Whereas rotation of $\phi_{I}$ induces CT from the I-ring to the P-ring in the gas-phase, rotation of $\phi_{P}$ induces $\mathrm{CT}$ from the P-ring to the I-ring.

Using ultrafast fluorescence and polarization spectroscopy experiments, ${ }^{27,}{ }^{36-37}$ Meech and coworkers identified similar short (hundreds of femtoseconds) and long (picoseconds) decay components for $\mathrm{HBDI}^{-}$across a variety of solvents. In water, time constants of $210 \mathrm{fs}$ and $1.1 \mathrm{ps}$ were reported for fluorescence decay — significantly faster than the 1.3/11.5 ps excited-state lifetimes reported in vacuo. ${ }^{21,30}$ Early quantum chemical calculations of gas-phase $\mathrm{pHBI}^{-38}$ suggested that a volumeconserving "hula-twist" deactivation mechanism ${ }^{39}$ is responsible for the limited sensitivity of the chromophore's excited-state lifetime on solvent viscosity. However, more recent in silico experiments implicate drastic torsional motion along either of the methine bridge bonds as responsible for the ultrafast deactivation of the anionic chromophore. ${ }^{34,40}$ This behavior is corroborated by high-level quantum mechanics/molecular mechanics $(\mathrm{QM} / \mathrm{MM})$ simulations in water using extended multistate multireference second-order perturbation theory (XMS-CASPT2) ${ }^{41}$ and spin-flip time-dependent density functional theory. ${ }^{42}$ These studies found that the dominant decay pathway of $\mathrm{pHBI}^{-}$is through the Itwisted channel. However, the limited simulation time considered (1 ps) did not allow identification of the sources responsible for the multi-timescale decay in solution. To resolve this, we simulate multiple picoseconds of the aqueous $\mathrm{HBDI}^{-}$photodynamics while monitoring important geometric and electronic signatures of the system.

Ultrafast dispersed pump-dump-probe experiments of $\mathrm{HBDI}^{-}$in water present excited-state decay lifetimes in the range 620-940 fs for twisted excited-state intermediates. ${ }^{43}$ Although the imperfect selectivity of a dump pulse may make it difficult to attribute time constants to specific processes, the $Z / E$ isomerization quantum yield derived from these experiments was below the detection threshold of $\sim 5 \%$. While torsion along $\phi_{I}$ can result in the $E$-isomer (Z/E isomerization), torsion along $\phi_{P}$ will regenerate the $Z$-isomer due to the symmetry of the P-ring ( $Z / Z$ isomerization). Thus, $Z / Z$ isomerization products are experimentally indistinguishable from decay pathways that do not involve isomerization. For this reason, it remains unclear what ratio of excited-state species proceeds toward photoproduct formation ( $Z / E$ isomerization), undergoes P-ring flip ( $Z / Z$ isomerization), or re-populates the original ground state 
without torsion (Z-isomer). A key ingredient of QM/MM (or fully QM) simulations of photochemistry is the proper description of the potential energy surface topography around conical intersections (CIs). Many electronic structure methods are not capable of correctly describing $\mathrm{S}_{0} / \mathrm{S}_{1}$ CI regions ${ }^{44-46}$ (e.g. time-dependent density functional theory and other single-reference methods). Following our recent gasphase study, ${ }^{24}$ we use the $\alpha$-CASSCF method, ${ }^{47}$ which provides a correct and efficient description of the exact degeneracy and relative energetics along key deactivation coordinates. This enables us to extend our simulations to multiple picoseconds. Consequently, we are able to monitor the chromophore's approach toward the CI seams and provide predictions for the photoproduct branching. The shape of conical intersections (e.g. peaked vs. sloped) can also be critically important in determining the photoproducts. ${ }^{48-51}$ Although it appears that the CIs become more peaked upon solvation for the neutral GFP chromophore ${ }^{52-53}$ (suggesting a shortening of the excited-state lifetime and a larger isomerization quantum yield), the shape of the CIs that govern nonadiabatic transitions for HBDI- in water has been largely unexplored.

Recent femtosecond stimulated Raman spectroscopy (FSRS) and transient absorption experiments of aqueous $\mathrm{HBDI}^{-31}$ fill in some of the gaps left by ultrafast fluorescence experiments. While time-resolved fluorescence experiments elucidate the temporal signatures of highly fluorescent HBDI $^{-}$species, i.e., closer to the Franck-Condon (FC) point, FSRS is able to track the progression of HBDI $^{-}$along vibrational modes of dark, non-fluorescent species. FSRS studies yield three time constants for the excited-state dynamics of HBDI: $\sim 350 \mathrm{fs}, 2-3 \mathrm{ps}$, and $\sim 250 \mathrm{ps}^{31}$ These were tentatively assigned to the formation of a charge-separated (CS) species, the transition from the CS state to a TICT state, and the ground-state recovery of the $Z$-isomer, respectively.

In this work, we explore the internal conversion dynamics following photoexcitation of explicitly-solvated $\mathrm{HBDI}^{-}$. Using ab initio multiple spawning ${ }^{54-58}$ (AIMS), we provide insight into the primary deactivation pathways in aqueous solution and each pathway's relative accessibility to CIs. In particular, we investigate the characteristics of $\mathrm{HBDI}^{-}$(i) at the $\mathrm{FC}$ region, (ii) out of the $\mathrm{FC}$ region as the molecule approaches CI seams, (iii) upon encountering the CI seam, and (iv) away from the CI seam as it returns to the ground state. This fully non-equilibrium behavior (chromophore and solvent response) is compared to the corresponding excited-state equilibrium solvation picture, defined by the free energy surface of aqueous $\mathrm{HBDI}^{-}$. Our results are interpreted in the context of previous experiments performed in silico, in vacuo, and in solution. Using this approach, we discuss the impact of solvation on $\mathrm{HBDI}^{-}$and develop a more complete picture of GFP chromophore photophysics.

Jones, List, Martínez - Photodynamics of solvated HBDI anion - Page 4 


\section{Computational Details and Methods}

Because of its efficiency and ability to describe CIs, state-averaged ${ }^{59}$ complete active space selfconsistent field (SA-CASSCF) ${ }^{60-62}$ was chosen as the electronic structure method for HBDI- in water. To increase the accuracy of SA-CASSCF, we correct for the lack of dynamic electron correlation in SACASSCF calculations by using $\alpha-$ CASSCF $^{47}$ (Figure S1). The $\alpha-C A S S C F$ method ${ }^{63}$ uses energy scaling to approximate the effects of dynamic electron correlation at the cost of traditional SA-CASSCF, similar to previous scaling methods introduced by Olivucci and coworkers. ${ }^{64}$ Orbitals are optimized to minimize the average energy of the three lowest singlet electronic states in SA-CASSCF, as this has been shown to provide an accurate excited-state description of I- and P-torsional pathways ${ }^{65}{ }^{6 n}$ particular, inclusion of three states captures the P-twisted minimum energy conical intersection (MECI) of $\mathrm{HBDI}^{-}$, which can be elusive when using a two-state averaging method with CASSCF. ${ }^{66}$ An active space of four electrons in three orbitals, i.e., SA3-CASSCF $(4,3)$, incorporates the bonding, non-bonding and antibonding $\pi$ orbitals relevant to describe the allylic anionic character of the methine bridge (Figure 2). Unless stated otherwise, the $6-31 \mathrm{G}^{*}$ basis set ${ }^{67}$ is used throughout.

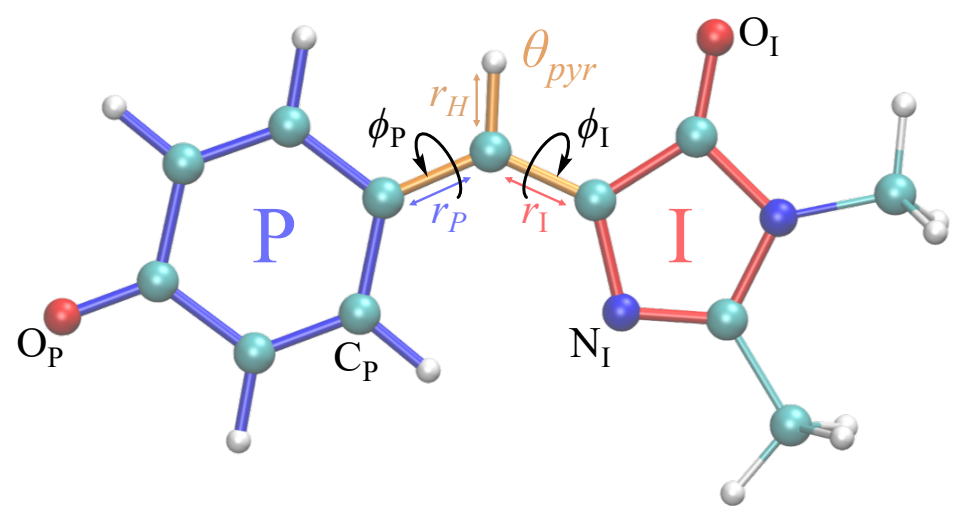

Figure 1. Structure of the HBDI- model chromophore illustrating defined geometric parameters. Cyan atoms represent carbon, red atoms represent oxygen, blue atoms represent nitrogen, and white atoms represent hydrogen. Blue, red, and orange bonds connect atoms belonging to the P-ring, I-ring, and methine bridge, respectively. $\phi_{I}$ is defined as the dihedral angle formed by $\mathrm{N}_{\mathrm{I}}$ and the carbon atoms of the methine bridge. $\phi_{P}$ is defined as the dihedral angle formed by the carbon atoms of the methine bridge and Cp. The pyramidalization of the central methine carbon atom, $\theta_{\mathrm{pyr}}$, is calculated as $\cos ^{-1}\left(\left(\vec{r}_{P} \times \vec{r}_{I}\right) \cdot \vec{r}_{H}\right)-\pi / 2$ and uses the bridge bond vectors in a method similar to Radhakrishnan et al. ${ }^{68}$ For reference, the pyramidalization angle of ideal $\mathrm{sp}^{2}$ and $\mathrm{sp}^{3}$ hybridized carbon atoms using this definition are $0^{\circ}$ and $55^{\circ}$, respectively. 

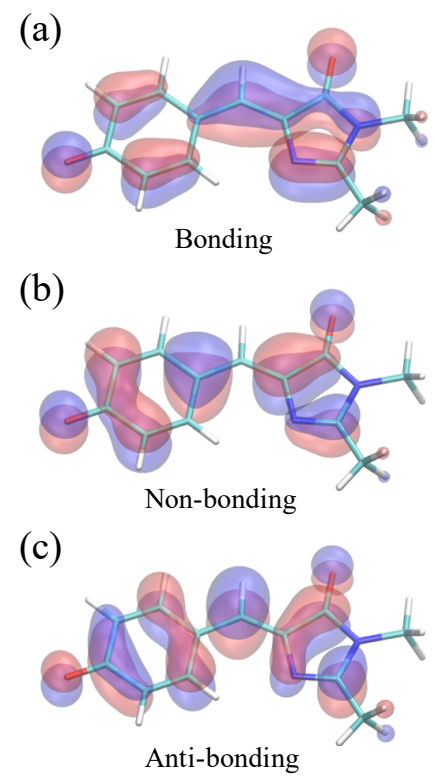

Figure 2. Active space orbitals of $\mathrm{HBDI}^{-}$from $\alpha(0.67)-\mathrm{SA} 3-\mathrm{CASSCF}(4,3)$ at the FC point: the anionic, allylic (a) bonding, (b) non-bonding, and (c) anti-bonding orbitals.

The $\alpha$ parameter was chosen to reproduce the $S_{0} \rightarrow S_{1}$ vertical excitation energy of the planar chromophore in solution, computed with the SA3-XMS-CASPT2 $(4,3)$ method $^{69-71}$ (1s orbitals of heavy atoms were excluded from the perturbation treatment and a level shift of 0.3 a.u. was used). This led to an $\alpha$ value of 0.67 for hydrated HBDI- (Figure S1). This is very similar to the $\alpha$ value of 0.64 which we found for isolated $\mathrm{HBDI}^{-}$(used in the gas-phase calculations reported herein). ${ }^{24}$ As shown in Figure S1, this $\alpha$-CASSCF method reproduces XMS-CASPT2 potential energy profiles along the two bridge torsional coordinates $\left(\phi_{I}\right.$ and $\left.\phi_{P}\right)$. Unless otherwise stated, all calculations were performed with $\alpha(0.67)$ SA3-CASSCF $(4,3) / 6-31 \mathrm{G}^{*}$ in explicit solvent. Relative energies and geometric parameters of representative optimized geometries are provided in the Supplementary Information (Figures S1-S3, Tables S1-S3). Fractional occupation number Hartree-Fock (FON-HF) was used to determine initial guess orbitals for the $\alpha$-CASSCF calculations. ${ }^{72-75}$ In the FON-HF implementation, energy levels were Gaussian-broadened using temperatures of 0.2 and 0.3 a.u. for gas-phase and solution-phase systems, respectively.

Critical points were obtained using the DL-FIND geometry optimization library. ${ }^{76}$ Electronic structure calculations were performed with TeraChem, ${ }^{77-81}$ exploiting graphic processing units.

Solvated initial conditions. A Boltzmann sampling procedure provided initial conditions (ICs) for the solvated chromophore. Using PACKMOL, ${ }^{78} \mathrm{HBDI}^{-}$was immersed in a solvent sphere comprised of 2000 water molecules and a sodium counterion. The radius of the solvation sphere was $24.3 \AA$, 
consistent with the density of liquid water $(0.997 \mathrm{~g} / \mathrm{mL})$. Classical dynamics were run with OpenMM, ${ }^{82}$ and a restraining spherical force of $10 \mathrm{kcal} / \mathrm{mol} / \AA^{2}$ was applied to the water sphere during the dynamics. The solvent was equilibrated by performing a 5 ns NVT classical dynamics simulation, using a Langevin thermostat at $300 \mathrm{~K}$, with the geometry of the chromophore fixed. Water molecules were treated with the flexible SPC/Fw model, ${ }^{83}$ and the sodium ion was modelled using van der Waals parameters previously determined for use in SPC/E water. ${ }^{84}$ Atomic charges for the chromophore were fit using the restrained electrostatic potential ${ }^{85}$ (RESP) procedure with HF/6-31G*, based on the B3LYP-D3/6-31G** optimized geometry of the chromophore in the gas-phase. ${ }^{86-88}$ Van der Waals parameters for the chromophore were taken from the general Amber force field (GAFF). ${ }^{89}$ Other force field parameters (such as equilibrium bond lengths/angles and associated force constants) are irrelevant because the geometry of the chromophore was kept fixed during the MM simulations used to relax the solvent environment.

Following the initial solvent equilibration, the geometric constraints on the chromophore were lifted and ground-state QM/MM molecular dynamics (QM/MM-MD) within the NVT ensemble were run for $10 \mathrm{ps}$ to obtain ICs for the nonadiabatic dynamics simulations. The chromophore was treated at the QM level (described above), whereas the waters and sodium ion were treated at the MM level. A D3 dispersion correction was included for the QM region, using parameters corresponding to HartreeFock. ${ }^{88}$ Spherical boundary conditions were again employed to avoid evaporation of water molecules. QM/MM-MD simulations were performed with a Langevin thermostat at $300 \mathrm{~K}$, using the timereversible Niklasson integrator ${ }^{90}$ and a time step of $0.5 \mathrm{fs}$. After an equilibration period of $3 \mathrm{ps,} \mathrm{a} \mathrm{total} \mathrm{of}$ 500 temporally-equidistant samples were extracted from the remaining 7 ps of QM/MM-MD to generate an absorption spectrum. The stick spectra were convolved with a Gaussian lineshape function, using a full width at half maximum (FWHM) of $0.40 \mathrm{eV}$ based on solution-phase ${ }^{91}$ experiments.

Nonadiabatic dynamics. A total of 50 ICs were extracted from the 500 QM/MM-MD samples (every tenth IC) and used to initiate AIMS simulations within the independent first generation approximation. ${ }^{57-58}$ Using this sampling procedure, we minimize the correlation between ICs. Moreover, this sampling procedure enables us to partition ICs based on their location in the aqueous HBDIabsorption spectrum, which is necessary to investigate the wavelength dependence of excited-state deactivation. ICs are classified as either red-shifted or blue-shifted based on their excitation energy relative to the computed absorption maximum (computed as $2.84 \mathrm{eV}$, compared to the measured value of $\left.2.91 \mathrm{eV}^{31,91}\right)$. When comparing our simulations to experiment, it should be noted that the wavelength 
of the pump used in previous time-resolved spectroscopy experiments ${ }^{31,92}$ was on the blue-edge of the spectrum (400 nm, i.e., $0.2 \mathrm{eV}$ blue-shifted from the absorption maximum). The AIMS simulations were continued for 5 ps or until at least $99 \%$ of the excited-state population for a given initial trajectory basis function (TBF) was transferred to the ground state. If a TBF on $\mathrm{S}_{0}$ went at least 5 fs without coupling with another TBF (as measured by the TBF overlap, with a threshold of 0.6), the TBF on $\mathrm{S}_{0}$ was removed from the set of coupled TBFs and run independently for the remainder of the simulation. The threshold for spawning (based on the dot product of the nonadiabatic coupling vector and the velocity) was defined as 0.005 a.u. The spherical boundary conditions applied for QM/MM-MD were employed during the AIMS dynamics as well. Error bars for exponential fitting of the $\mathrm{S}_{1}$ population were obtained using a bootstrapping method ${ }^{93}$ that included 1000 bootstrapping samples. To gauge the branching ratio of photoproducts from AIMS dynamics, each spawned TBF on $\mathrm{S}_{0}$ was propagated independently for an additional $500 \mathrm{fs}$. By the end of the extended ground-state dynamics, the TBFs were classified based on the $\phi_{\mathrm{I}}$ and $\phi_{\mathrm{P}}$ values of their $\mathrm{S}_{0}$ nuclear configuration. If the magnitude of either dihedral was above $120^{\circ}$, the product was classified as isomerized with respect to that angle. The resulting photoproducts were used to quantify photoisomerization quantum yield. Geometric analysis of AIMS trajectories was aided by MDTraj. ${ }^{94}$

Umbrella sampling. To provide insight regarding the free energy landscape and entropic effects of $\mathrm{HBDI}^{-}$in water, QM/MM umbrella sampling at $300 \mathrm{~K}$ was performed on $\mathrm{S}_{1}$. A two-dimensional mapping was performed along the $\phi_{\mathrm{I}}$ and $\phi_{\mathrm{P}}$ twisting coordinates (ranging from $-120^{\circ}$ to $120^{\circ}$, using $10^{\circ}$ intervals for a total of 625 sampling windows). To compensate for incomplete sampling of solvent configurations, we augmented the data to incorporate the symmetry with respect to twisting of the $\phi_{\mathrm{I}}$ and $\phi_{\mathrm{P}}$ dihedral angles (Figure S4). Specifically, each data point (i.e., energy, oscillator strength) for an observed geometry contributed to two data points: one for the observed geometry and one for its

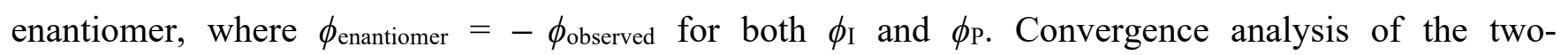
dimensional free energy surface indicated that an equilibration time of $1.5 \mathrm{ps}$ for each window provided a sufficient compromise between sampling and computational cost (Figure S5). After the equilibration, 2 ps of data was collected for each window to generate the potential of mean force ${ }^{95}$ (PMF). In total, over 2 ns of excited-state QM/MM-MD simulations were run to obtain the free energy surface. A harmonic biasing potential with a force constant of $100 \mathrm{kcal} / \mathrm{mol} / \AA^{2}$ was applied to both dihedrals. Contour plots were generated using a cubic interpolation between points on the free energy grid obtained from unbiasing a set of biased sampling windows via the weighted histogram analysis method. ${ }^{96-97}$

Jones, List, Martínez - Photodynamics of solvated HBDI anion - Page 8 
Time-resolved fluorescence. Two-dimensional fluorescence spectra of HBDI- were generated based on the AIMS simulations. Spectra were obtained as:

$$
\mathrm{F}(\mathrm{t}, v) \propto \sum_{\mathrm{I} \in \mathrm{S}_{1}}^{N_{\text {TBFs }}} \mathrm{n}_{\mathrm{I}}(\mathrm{t})\left|\mu_{\mathrm{S}_{0} / \mathrm{S}_{1}}\left(\overline{\mathrm{R}}_{I}(\mathrm{t})\right)\right|^{2}\left(\frac{\Delta \mathrm{E}_{\mathrm{S}_{0} / \mathrm{S}_{1}}\left(\overline{\mathrm{R}}_{I}(\mathrm{t})\right)}{\mathrm{h}}\right)^{3} \delta\left(\Delta \mathrm{E}_{\mathrm{S}_{0} / \mathrm{S}_{1}}\left(\overline{\mathrm{R}}_{I}(\mathrm{t})\right)-\mathrm{h} v\right)
$$

where the sum is restricted to TBFs that are propagating on $\mathrm{S}_{1}, \mathrm{~h}$ is Planck's constant, $\mathrm{n}_{\mathrm{I}}(\mathrm{t})$ is the $S_{1}$ population of the $\mathrm{I}^{\text {th }} \mathrm{TBF}, \mu_{\mathrm{S}_{0} / \mathrm{S}_{1}}(R)$ is the transition dipole moment between $S_{0}$ and $S_{1}$ at molecular geometry $R, \Delta \mathrm{E}_{\mathrm{S}_{0} / \mathrm{S}_{1}}(R)$ represents the $\mathrm{S}_{0} / \mathrm{S}_{1}$ energy gap, and $\bar{R}_{I}(t)$ is the position centroid of the $I$ th TBF at time $t .^{98}$ The time-resolved fluorescence spectra were convolved with a Gaussian having FWHM of $0.15 \mathrm{eV}$ in the energy domain $(v)$ and $100 \mathrm{fs}$ in the temporal domain $(t)$ to simulate the instrument response function of previous ${ }^{27,99}$ fluorescence up-conversion experiments. For each two-dimensional fluorescence spectrum, the energy was shifted by $0.13 \mathrm{eV}$ to align the fluorescence intensity maxima obtained from theory and experiment (vide infra). ${ }^{31}$ Once shifted, the decay signal corresponding to an intensity slice at $500 \mathrm{~nm}$ was extracted and analyzed to elucidate the fluorescence behavior of HBDIover the course of the dynamics.

\section{Results and Discussion}

To begin describing the effects of solvation, we observe how the absorption spectrum of $\mathrm{HBDI}^{-}$ changes when the molecule is immersed in water. Figure 3a compares the simulated and experimental absorption spectra of $\mathrm{HBDI}^{-}$in vacuum ${ }^{24}$ and aqueous solution. Solvation of $\mathrm{HBDI}^{-}$blue-shifts the absorption maximum $(0.41 \mathrm{eV})$, as is also observed in experiment $(0.32 \mathrm{eV}) .{ }^{31,91,100}$ Radial distribution functions (RDFs), based on the ground-state QM/MM-MD, between water oxygen atoms and the heteroatoms in $\mathrm{HBDI}^{-}$provide a description of local solvation. As shown in Figure $3 \mathrm{~b}$, the RDF for the $\mathrm{O}_{\mathrm{P}}$ atom indicates that it is more tightly coordinated to water than the $\mathrm{O}_{\mathrm{I}}$ atom. This is consistent with the enhanced charge localization on $\mathrm{O}_{\mathrm{P}}$, relative to $\mathrm{O}_{\mathrm{I}}$, in the ground state (see Figure S6 for fragmentaccumulated Mulliken charges). 

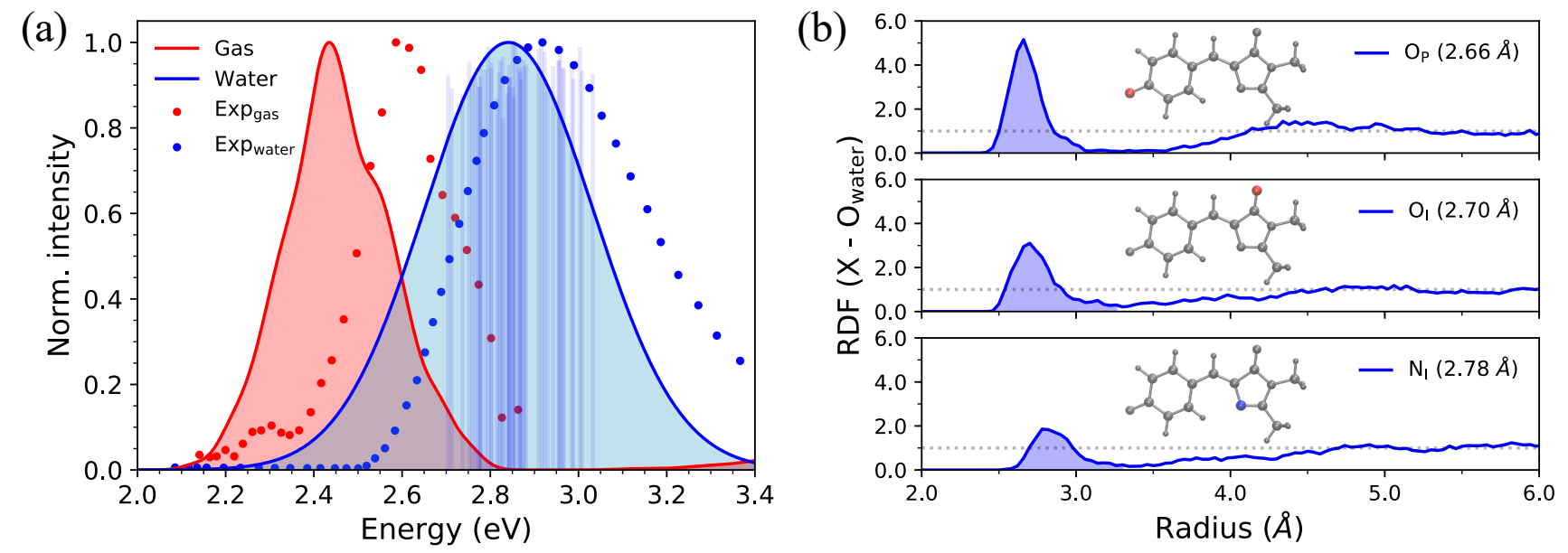

Figure 3: (a) Simulated absorption spectra of $\mathrm{HBDI}^{-}$in gas-phase (red) and water (blue) using 500 ICs from each system. Sticks represent the $\mathrm{S}_{0} / \mathrm{S}_{1}$ excitation energy and their relative oscillator strengths for the 50 solvated ICs used for AIMS simulations, at the $\alpha(0.67)-S A 3-C A S S C F(4,3) / 6-31 \mathrm{G}^{*}$ level. Individual sticks for gas-phase (FWHM of $0.07 \mathrm{eV}$ ) and aqueous (FWHM of $0.40 \mathrm{eV}$ ) systems were convolved with a Gaussian envelope based on experimental FWHM. Simulated gas-phase data was taken from List et al. ${ }^{24}$ sampling from a 300K harmonic Wigner distribution and using $\alpha(0.64)-\mathrm{SA} 3-$ CASSCF $(4,3) / 6-31 G^{*}$. Experimental gas-phase and solvated chromophore spectra were obtained by digitizing data from Nielsen et al. ${ }^{100}$ and Andersen et al. ${ }^{91}$ respectively. (b) Ground-state radial distribution functions for heteroatoms in $\mathrm{HBDI}^{-}$ (highlighted in each panel and identified in Figure 1) with water oxygen atoms. Shaded regions represent the first solvation shell.

Pinpointing the origin of this blue shift enables a more in-depth understanding of the changes that take place once the chromophore is solvated. To isolate the geometric and electronic perturbations induced by explicit solvation, we observe the shift of the absorption spectrum based on whether HBDI $^{-}$ is isolated or surrounded by water. In particular, a comparison of the QM/MM-MD absorption spectrum of aqueous $\mathrm{HBDI}^{-}$to that generated by the same $\mathrm{HBDI}^{-}$configurations but in isolation allows us to directly gauge the electronic effects of solvation. Similarly, the spectral shift obtained by comparing the absorption spectrum of the solvent-removed configurations to that from a gas-phase QM/MM-MD sampling (Figure S7) enables us to isolate geometric effects of the solvent. As summarized in Table 1, the electronic properties of water are primarily responsible for blue-shifting the absorption maximum $\left(\Delta \mathrm{E}_{\text {abs }}\right)$ of $\mathrm{HBDI}^{-}$upon solvation $(0.19 \mathrm{eV})$, whereas the geometric changes have a minimal effect $(0.03$ $\mathrm{eV})$. 
Table 1: Change in the absorption maximum of $\mathrm{HBDI}^{-}$as a function of the chromophore's environment. The calculated $\Delta \mathrm{E}_{\text {abs }}$ from ground-state $\mathrm{QM} / \mathrm{MM}-\mathrm{MD}$ sampling, either in the gas-phase or in water, is compared to the $\Delta \mathrm{E}_{\text {abs }}$ calculated for configurations of aqueous HBDI- following the removal of the solvent. The same solvent-removed samples are used for comparison to gas-phase and water QM/MM-MD samples. However, since $\alpha$ is a scaling parameter, choosing an $\alpha$ value consistent with the QM/MM-MD sampling procedure is necessary to avoid spurious energy shifts upon comparison.

\begin{tabular}{|c|c|c|c|}
\hline & $\begin{array}{c}\Delta \mathbf{E}_{\text {abs }} \text { maximum: } \\
\mathbf{Q M} / \mathbf{M M - M D}(\mathbf{e V})\end{array}$ & $\begin{array}{c}\Delta \mathbf{E}_{\text {abs }} \text { maximum: } \\
\text { Solvent removed (eV) }\end{array}$ & $\begin{array}{c}\Delta \Delta \mathbf{E}_{\text {abs }} \\
\text { maximum: }\end{array}$ \\
\hline Gas $(\boldsymbol{\alpha}=\mathbf{0 . 6 4})$ & $2.56 \pm 0.07$ & $2.53 \pm 0.07$ & 0.03 \\
\hline Water $(\boldsymbol{\alpha}=\mathbf{0 . 6 7})$ & $2.84 \pm 0.09$ & $2.65 \pm 0.08$ & 0.19 \\
\hline
\end{tabular}

To understand the limited impact of geometric perturbations, we analyze the initial distribution of key geometric parameters to observe how the sampled configuration space changes upon solvation. Figure $4 \mathrm{a}$ shows the bond length distributions for the bridge bonds, $r_{I}$ and $r_{P}$, from the ground-state QM/MM-MD. In water, the bridge bonds of $\mathrm{HBDI}^{-}$sample a narrower region of configuration space when compared to vacuum. This difference is related to whether a quantum or classical sampling technique is used, as indicated by the similar configuration space sampled by gas-phase and solutionphase HBDI- using QM/MM-MD sampling in both instances (Figure S7). Distributions for the solvated ICs used for the AIMS dynamics are shown in Figure S8. The average $r_{I}$-to- $r_{P}$ bond length ratio in water is consistent with a stabilization of the phenolate resonance structure, in line with the RDFs in Figure $3 \mathrm{~b}$. Therefore, we verify that the phenolate resonance structure of $\mathrm{HBDI}^{-}$is favored throughout the groundstate dynamics in water, as postulated by calculations in a polarizable continuum water model. ${ }^{39}$ While the phenolate and quinoid resonance structures contribute almost equally in gas-phase, ${ }^{24,65}$ the decrease in the $r_{I}$-to- $r_{P}$ ratio upon solvation indicates a preference toward the phenolate resonance structure in solution. Interestingly, the distributions along the $\phi_{\mathrm{I}}$ and $\phi_{\mathrm{P}}$ dihedrals (Figure $4 \mathrm{~b}$ ) remain almost identical between systems. Both systems have broad dihedral angle distributions, with values ranging from approximately $-30^{\circ}$ to $+30^{\circ}$, centered about zero degrees. Disrotatory motion, i.e., the concerted rotation of $\phi_{I}$ and $\phi_{P}$ in opposite directions, conserves angular momentum in both ground-state systems. The striking similarity between these angular distributions indicates that the presence of mobile water molecules does not restrict the chromophore's motion along its twisting dihedrals on the ground state. 

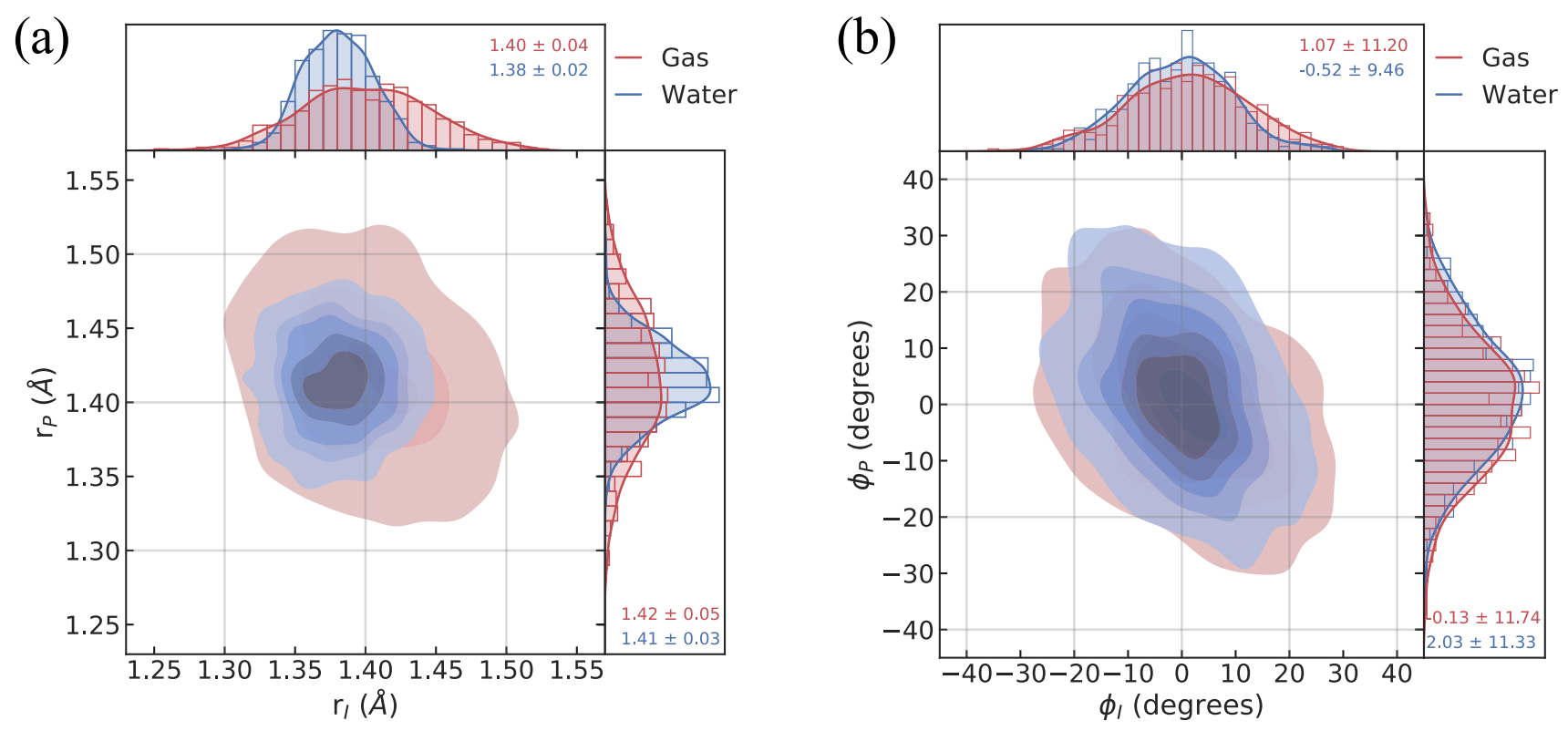

Figure 4: Geometric characterization of ICs using the ground-state distributions of (a) carbon-carbon methine bridge bonds adjacent to the imidazolinone $\left(r_{I}\right)$ and phenolate $\left(r_{P}\right)$ rings and (b) dihedral angles associated with imidazolinone $\left(\phi_{I}\right)$ and phenolate $\left(\phi_{P}\right)$ twisting. Red indicates data for ICs sampled from gas-phase harmonic Wigner distribution at $300 \mathrm{~K}$. Blue indicates data for ICs sampled from aqueous QM/MM-MD simulations at 300K (via a Boltzmann sampling procedure).

Electronic effects of equilibrium solvation. Before investigating the photodynamics of $\mathrm{HBDI}^{-}$ out of the FC region, we start by considering the excited-state landscape in the equilibrium solvation regime. Although the difference in time scales of $\mathrm{HBDI}^{-}$intramolecular vibrations and solvent relaxation (e.g. time constants of 0.16 and $1.2 \mathrm{ps}$ were reported for solvent relaxation following photoexcitation of a coumarin dye ${ }^{101}$ ) means that an equilibrium picture is unlikely to pertain to the dynamics induced by photoexcitation, an excited-state PMF identifies the energetically favored configurations of aqueous $\mathrm{HBDI}^{-}$following excitation. In other words, the PMF indicates the stability of twisted $\mathrm{HBDI}^{-}$structures that are surrounded by (partially or fully) relaxed solvent configurations. Such insight is useful for categorizing stable excited-state intermediates and interpreting the photodynamics of aqueous $\mathrm{HBDI}^{-}$.

From two-dimensional umbrella sampling on $\mathrm{S}_{1}$, along the bridge dihedrals, Figure 5 shows that single-bond twisting about either of the bridge bonds is favored. The resulting PMF highlights four energetic wells located near $90^{\circ} \mathrm{I}$ - and P-twisted structures on $\mathrm{S}_{1}$. Interestingly, the excited-state dynamics do not follow the minimum energy path to reach these energetic wells (vide infra). Instead, the chromophore reaches highly-twisted configurations by following either the I- or P-channels illustrated in Figure 5.

To separate out the electronic effects of equilibrium solvation, Figure 6 displays the average $\mathrm{S}_{0} / \mathrm{S}_{1}$ energy gap across all windows in solvent and solvent-stripped configurations. In water (Figure 6a), 
the observed minima along $\phi_{\mathrm{I}}$ lie close to the CI seam, whereas rotation along $\phi_{\mathrm{P}}$ leads to minima on $\mathrm{S}_{1}$ that are $\sim 1 \mathrm{eV}$ removed from $\mathrm{S}_{0}$. This suggests that the I-twisted CI seam is more peaked than the Ptwisted CI seam. The absence of electronic degeneracy associated with P-twisting hints that energetically and/or entropically unfavorable geometric changes are required to reach degeneracy along this pathway. Figure $6 \mathrm{~b}$ shows that I-twisted intermediates have lower $\mathrm{S}_{0} / \mathrm{S}_{1}$ energy gaps than those of $\mathrm{P}$ twisted intermediates in the gas-phase, as observed in solution. This energetic asymmetry originates from the differences in electron affinity between the I- and P-rings. ${ }^{24}$ However, to conclude whether or not water stabilizes one twisted structure over the other, it is necessary to look at the energetic differences caused by solvation. The difference between water and gas-phase energy gaps (Figure S9) reveals that water introduces non-selective gap reduction $(\sim 0.3 \mathrm{eV})$ for highly-twisted $\left(\sim 90^{\circ}\right)$ structures - the I- and P-twisted energy gaps decrease by approximately the same amount upon solvation. Yet, as previously discussed for Figure $6 \mathrm{a}$, the consequences of this gap reduction are significant. This equilibrium solvation picture suggests that (i) aqueous $\mathrm{HBDI}^{-}$proceeds along one-bond-flip-dominated coordinates toward the twisted CI seam, as in gas-phase, ${ }^{24}$ (ii) the I-twisted CI seam is more accessible than its P-twisted counterpart, and (iii) solvation promotes internal conversion through the I-twisted CI seam, similar to its neutral counterpart. ${ }^{52-53}$

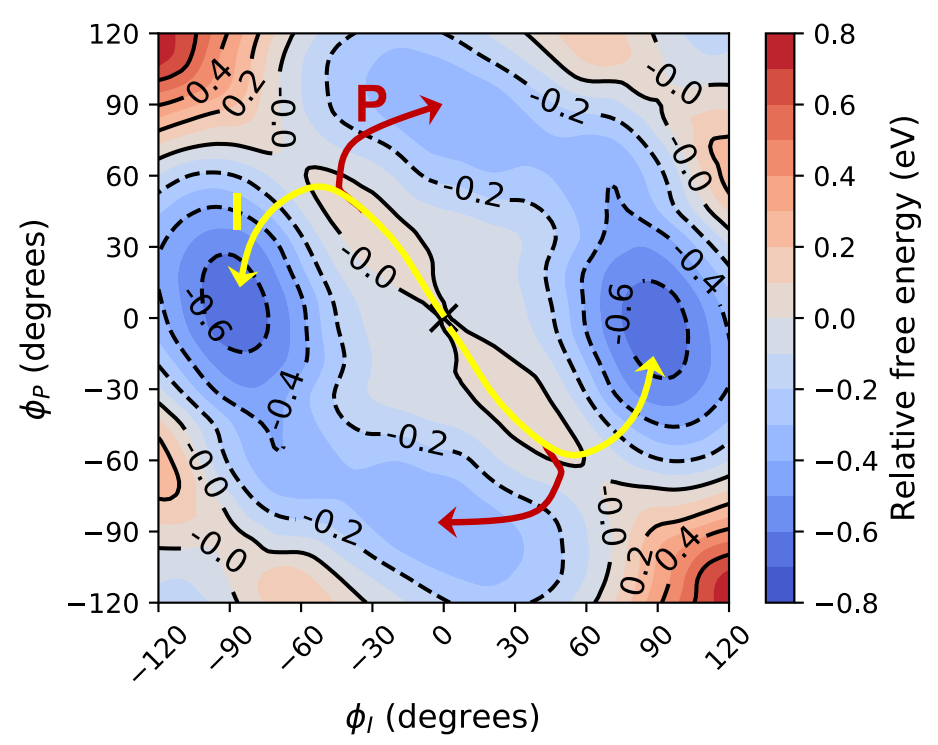

Figure 5: Relative free energy profile on $\mathrm{S}_{1}$ obtained from two-dimensional umbrella sampling along $\phi_{I}$ and $\phi_{P}$. The zero point (marked with an "X") corresponds to the planar structure. The observed deactivation pathways through I-twisting (yellow) and P-twisting (red) are depicted schematically. 

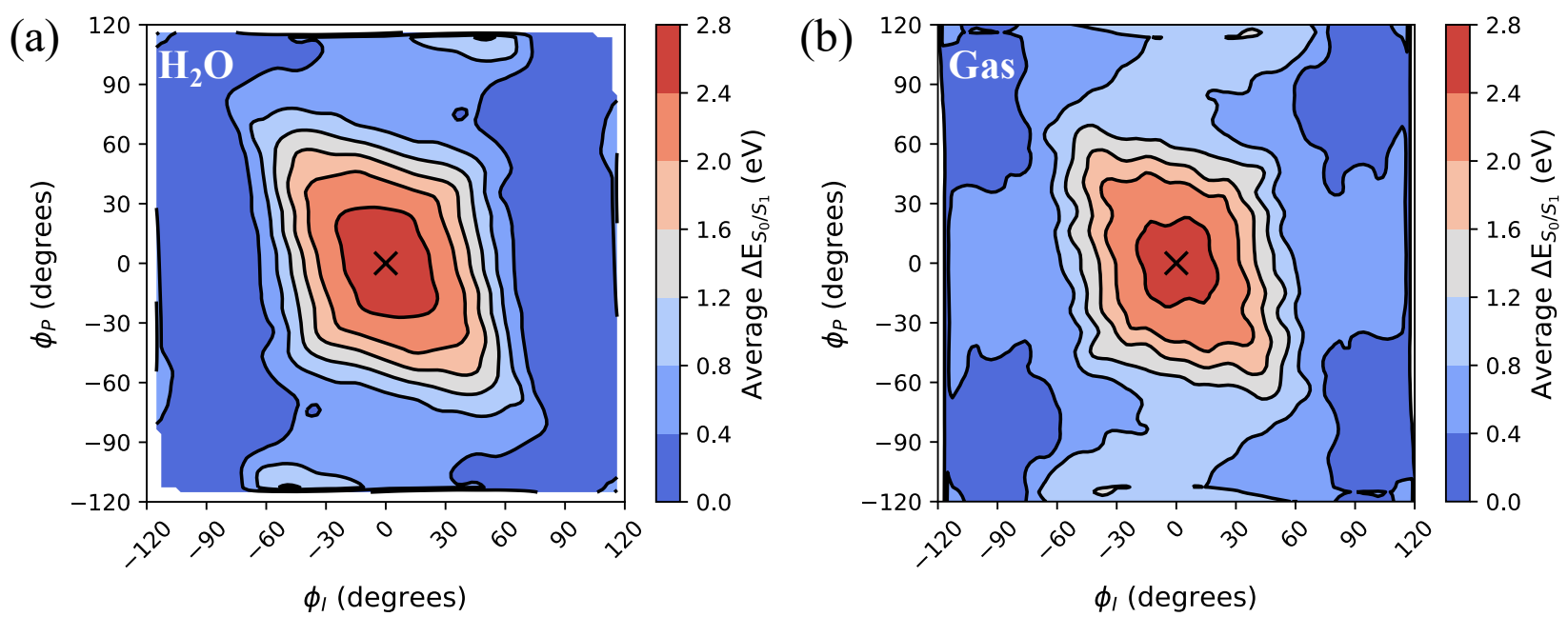

Figure 6: Contour plot of the average $\mathrm{S}_{0} / \mathrm{S}_{1}$ energy gap with respect to $\phi_{I}$ and $\phi_{P}$ dihedral angles (a) in water and (b) in gasphase. Gas-phase energies correspond to solvated structures (5000 points total, 8 per window) from (a) after removing the solvent around the chromophore. The average dihedral angles and energy gaps were plotted for each $10^{\circ}$ window from the umbrella sampling, and cubic interpolation was performed between these points. The zero point (marked with an "X") corresponds to the planar structure. All energies were computed with $\alpha(0.67)-\operatorname{SA} 3-\operatorname{CASSCF}(4,3) / 6-31 \mathrm{G}^{*}$.

Nonadiabatic dynamics of HBDI- in water. Shifting into the non-equilibrium regime (i.e., the solvent does not have enough time to relax around the chromophore), we analyze the behavior of aqueous HBDI- following photoexcitation. As shown by the $\mathrm{S}_{1}$ population decay in Figure 7 , over half of the excited-state population is transferred to the ground state within the first $1 \mathrm{ps}$. Such a rapid decay is consistent with the ultrafast deactivation reported in experiments. ${ }^{27,31,43}$ Yet, despite the sub-ps deactivation, a fraction of the population remains on the excited state for $>5 \mathrm{ps}$. This biphasic pattern inspired a delayed bi-exponential fit to the decay profile:

$$
\mathrm{P}(\mathrm{t})=\operatorname{Aexp}\left(-\frac{\mathrm{t}-\tau_{0}}{\tau_{1}}\right)+(1-\mathrm{A}) \exp \left(-\frac{\mathrm{t}-\tau_{0}}{\tau_{2}}\right)
$$

where $t$ represents time, $A$ is a weighting coefficient, $\tau_{0}$ represents the time at which population transfer begins (lagtime), and $\tau_{1}$ and $\tau_{2}$ are characteristic lifetimes associated with the decay of the $S_{1}$ population over time, $\mathrm{P}(t)$. Constants obtained from this fitting procedure, through the use of bootstrapping, are: $A=$ $0.50 \pm 0.19, \tau_{0}=204 \pm 43 \mathrm{fs}, \tau_{1}=609 \pm 181 \mathrm{fs}$, and $\tau_{2}=3.1 \pm 1.2 \mathrm{ps}$. 


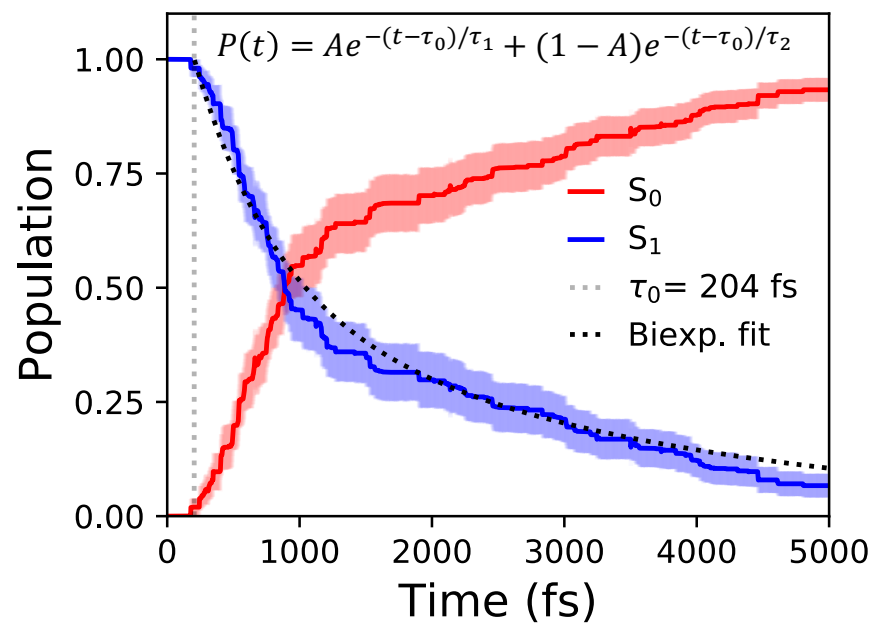

Figure 7: Ground- and excited-state populations during AIMS simulations. Fifty ICs were used in the explicitly-solvated system. The average state population is represented with solid lines. The vertical, gray dotted line represents the time at which population transfer begins. The black dotted line is the delayed bi-exponential fit to the $\mathrm{S}_{1}$ population decay. Error bars are illustrated using transparent lines.

Viewing the dynamics within defined time intervals reveals novel information regarding the time scales of each deactivation pathway available to aqueous $\mathrm{HBDI}^{-}$- two distinct sub-populations exist with different excited-state lifetimes (Figure 8). In accordance with the decreased electronic density along the methine bridge upon excitation (as illustrated in Figure 2) facile rotation along $\phi_{I}$ and $\phi_{P}$ is permitted on the excited state. Monitoring the $\mathrm{S}_{1}$ population over time, with respect to the $\phi_{I}$ and $\phi_{P}$ dihedrals, illustrates (i) the accessibility and (ii) the population transfer efficiency of each internal conversion avenue taken by the ICs. During the first $100 \mathrm{fs}$, the $\mathrm{S}_{1}$ wavepacket remains localized near the FC region. Rather than following the minimum energy path along one-bond-flip coordinates (Figure 5), the disrotatory motion exhibited during the ground-state dynamics (Figure 4b) is preserved. This indicates a non-equilibrium solvation regime where the response of the surrounding waters is too slow to impede disrotatory motion out of the FC region, which is otherwise associated with a small free energy barrier (less than $0.2 \mathrm{eV}$ ). Once the free energy along the disrotatory path becomes too large to overcome, the molecule reaches a fork in the road. Here, the wavepacket bifurcates via a process that involves a one-bond-flip-like rotation along either $\phi_{I}$ or $\phi_{P}$ - with one path leading to highly I-twisted structures (I-channel) and the other path leading to highly P-twisted structures (P-channel). Because this drastic dihedral torsion is preceded by disrotatory motion out of the FC region, the internal conversion process resembles an aborted hula-twist mechanism. The branching associated with this mechanism becomes apparent by $500 \mathrm{fs}$ as exploration into the I- and P-channels enables population transfer to the 
ground state. By 1 ps, motion along the torsional angles is more prominent as the I- and P-channels arise as the exclusive avenues for population transfer. Essentially no TBFs occupy near-planar configurations after 1.75 ps of dynamics, as four distinct regions in configuration space emerge as dihedral basins. These basins coincide with the energetic wells identified in Figure 5. Interestingly, 250 fs later, no evidence of the I-twisted species is present while the P-twisted species remains on $\mathrm{S}_{1}$ for several picoseconds. The disappearance of the I-twisted species on $\mathrm{S}_{1}$ is explained by an ultrafast population transfer to the ground state, permitted by the electronic degeneracy highlighted in Figure 6a. For this part of the wavepacket, twisting along $\phi_{I}$ is enough to induce population transfer to the ground state. The persistence of the P-twisted species for several picoseconds on the excited state signifies that dihedral torsion around $\phi_{P}$ alone is not sufficient to bring the $\mathrm{S}_{0}$ and $\mathrm{S}_{1}$ electronic states close enough together to facilitate efficient population transfer. Rather, additional geometric requirements are necessary for the Pspecies to participate in internal conversion.

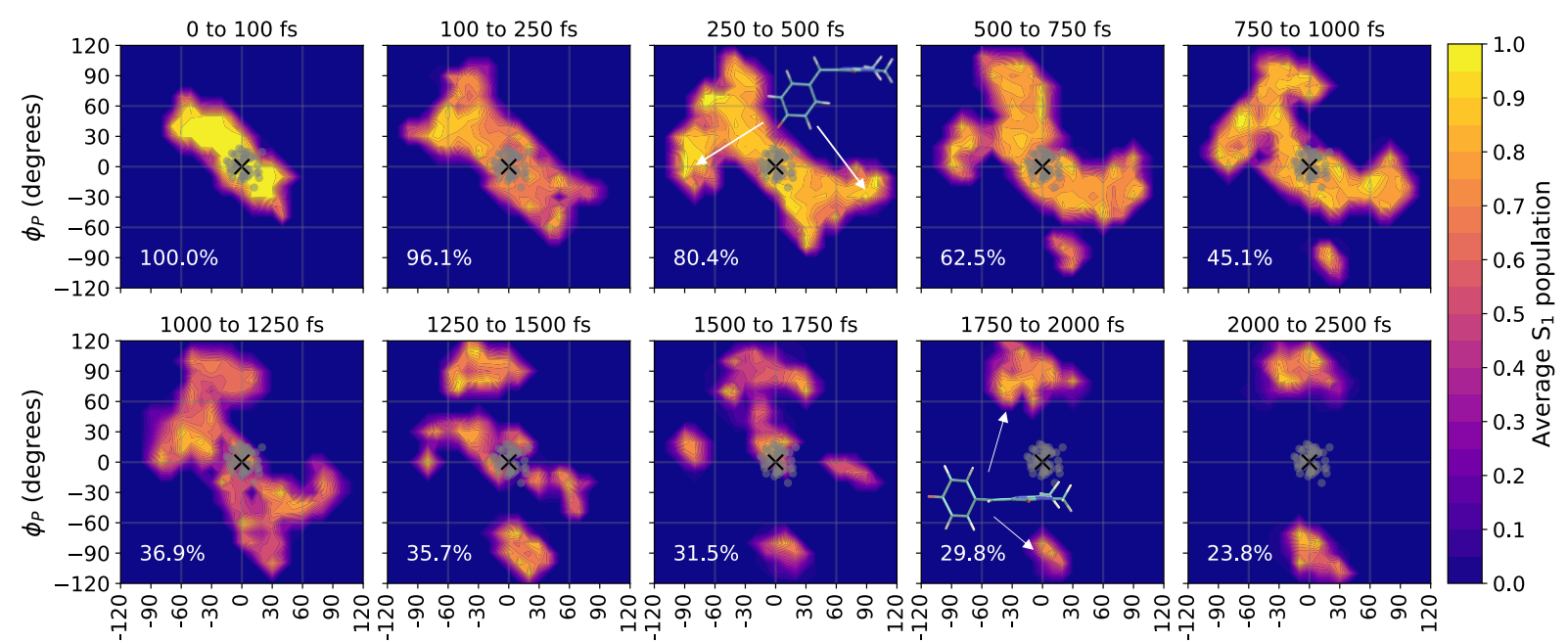
$\phi_{l}$ (degrees)
$\phi_{1}$ (degrees)
$\phi_{l}$ (degrees)
$\phi_{l}$ (degrees)
$\phi_{l}$ (degrees)

Figure 8: Time evolution of the reduced $S_{1}$ density along the bridge dihedrals during the first 2.5 ps of solution-phase AIMS dynamics. Average dihedral angles and $\mathrm{S}_{1}$ populations were computed using $10^{\circ}$ intervals, and linear interpolation was performed between these points. The percentages displayed in each panel represent the total $S_{1}$ population at the end of the specified time window, relative to their maximum at time $=0$. Gray dots indicate the dihedral angles of the fifty initial conditions.

The time scale differences established in Figure 8 were refined using the $\mathrm{S}_{1}$ population profiles for the I- and P-deactivating species (Figure 9). In the case of I-twisted deactivation, the entire excitedstate population is transferred to the ground state within $\sim 1.5 \mathrm{ps}$, with decay initiating at $\sim 175$ fs. This rapid initiation and conclusion of population transfer for the I-species is due to an easily accessible Itwisted CI seam that efficiently transfers population to the ground state, in accordance with Figure 6. On 
the other hand, the P-deactivation mechanism is associated with a species that persists on $\mathrm{S}_{1}$ for $\sim 540 \mathrm{fs}$ before population transfer begins. Furthermore, this mode of population transfer is much slower and less efficient, as $\sim 10 \%$ of the population remains on the excited state after 5 ps. A similar analysis compares the deactivation of red-shifted and blue-shifted ICs (Figure S10, Table S4).

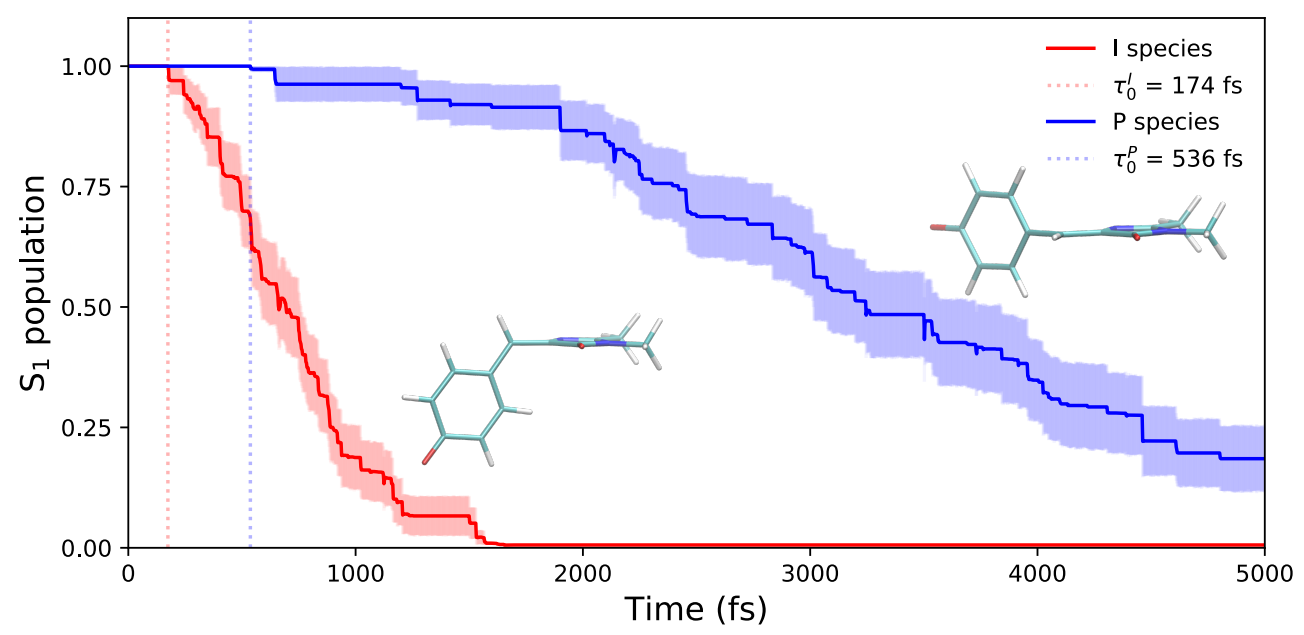

Figure 9: Excited-state population of $\mathrm{HBDI}^{-}$in water for I- and P-deactivating ICs. Solid lines represent the average populations for each species. Transparent lines represent the error bars obtained from bootstrapping analysis. We indicate the delay time before population transfer $\left(\tau_{0}^{I} / \tau_{0}^{P}\right)$ for each set with vertical dotted lines, emphasizing that deactivation is faster for I-twisting compared to P-twisting.

After identifying the characteristic twisting pathways, the dynamics out of the FC region were probed using various electronic and geometric parameters (Figure 10, Figure S11). Here, the parent TBFs are partitioned into I- and P-twisted species based on whether the I- or P-channel was taken, respectively. Our results corroborate that the dominant mode of deactivation $(66 \%)$ is through torsion along $\phi_{I}$, in agreement with nonadiabatic dynamics simulations at the XMS-CASPT2 ${ }^{41}$ and spin-flip time-dependent density functional theory ${ }^{42}$ levels. The violin plots in Figure 10 present the distribution of key electronic and geometric observables sampled across all parent TBFs of each species during the first 500 fs. Characteristic activity along the $\phi_{I}$ and $\phi_{P}$ twisting dihedrals is observed within the first 500 fs. As expected, the I-species samples along $\phi_{I}$ more aggressively than it does along $\phi_{P}$, and the opposite behavior is true for the P-species. Worth noting, however, is the time scale at which both species reach $\sim 90^{\circ}$ rotations of their respective dihedrals. Between 200 and $400 \mathrm{fs}$, both species begin to explore and populate these highly twisted configurations. As in the gas-phase, ${ }^{35}$ we observe that twisting along $\phi_{I}$ facilitates CT from the I-ring to the P-ring while twisting along $\phi_{P}$ facilitates $\mathrm{CT}$ from the P-ring to the I- 
ring. Furthermore, the molecule's rapid approach toward highly I- and P-twisted structures indicates that access to their respective TICT states are downhill in solution.

In the gas-phase, bond length alternation and bridge pyramidalization are the main coordinates used by HBDI- to reach the CI seam. ${ }^{24}$ As shown in Figure 10 (300 - 500 fs), displacement along the pyramidalization coordinate is attenuated for the I- and P-twisted species in solution relative to gasphase $\left(\left|\theta_{\text {pyr }}\right|\right.$ of $30^{\circ}$ and $48^{\circ}$ for I- and P-twisted MECIs, ${ }^{24}$ respectively). Because solvation essentially closes the energy gap of the I-twisted species but not completely for the P-twisted, the bridge pyramidalization requirement for reaching the I-twisted CI seam becomes almost negligible. Compared to species along the I-channel, larger-amplitude displacements of the pyramidalization coordinate are required to trigger population transfer along the P-channel. This suggests that the path to the I-twisted CI seam is essentially energetically downhill, while this process is energetically uphill for the P-twisted case.

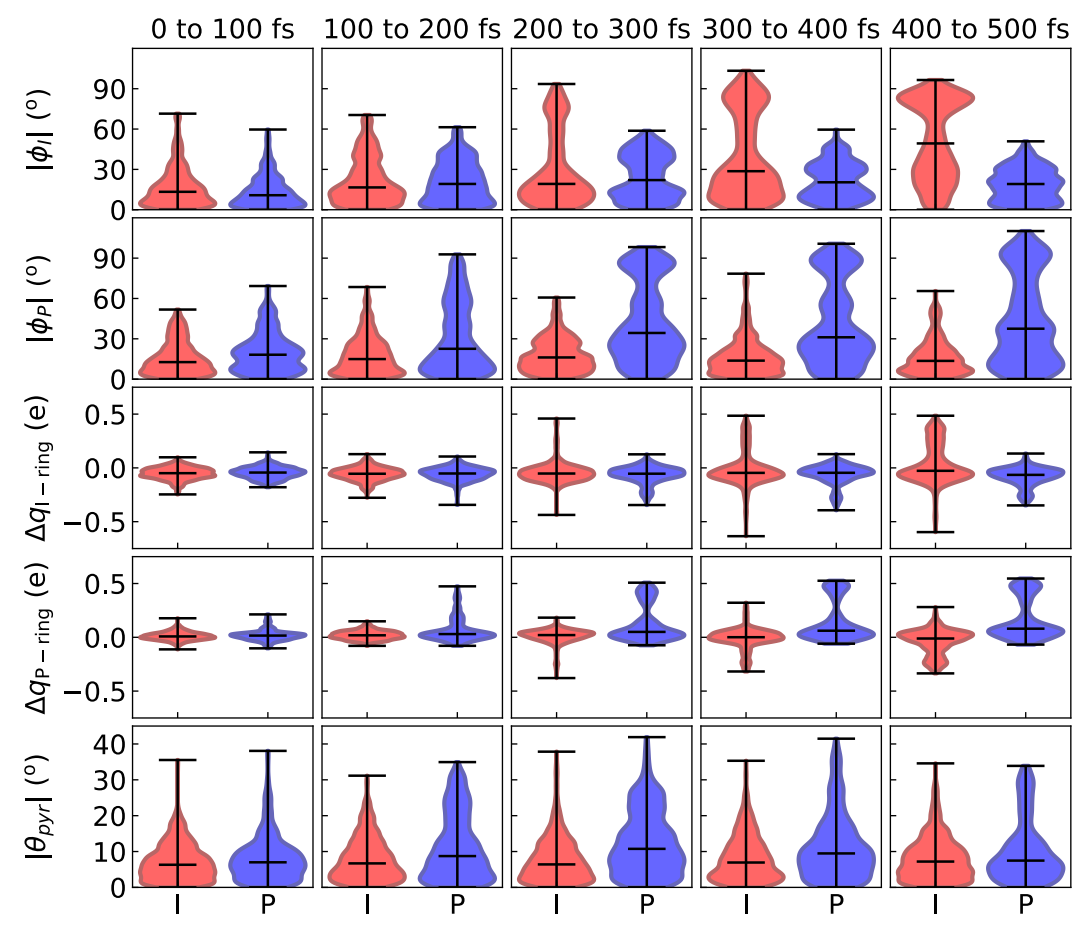

Figure 10: Violin plots that monitor density distributions of geometric and electronic observables out of the FC region for Ideactivating (red) and P-deactivating species (blue). Shown data monitors observables for the parent TBFs from each IC during the first $500 \mathrm{fs}$.

In addition to monitoring the geometric and electronic changes that occur during the first few hundred femtoseconds following photoexcitation, the response of the solvation environment during the first 1 ps was investigated. Figure 11 shows the evolution of the first solvation peak for the parts of the wavepacket following the I- and P-twisted pathways. In the case of the I-deactivating species in Figure 
11a, there are no significant changes to the RDF profiles of $\mathrm{O}_{I}$ or $\mathrm{N}_{\mathrm{I}}$ during the first 1 ps. However, the first solvation peak of $\mathrm{O}_{\mathrm{P}}$ experiences a slight decrease over time. This contrasts the evolution of RDFs for the same atoms in the P-deactivating species in Figure 11b. Over the first $1 \mathrm{ps}$, the maximum of the first solvation peak for $\mathrm{O}_{\mathrm{P}}$ and $\mathrm{O}_{\mathrm{I}}$ gradually decreases and increases, respectively, toward the RDFs associated with equilibrated $\phi_{P}$-twisted structures. This trend is consistent with the TICT behavior identified in Figure 10. Upon torsion along $\phi_{P}, \mathrm{CT}$ occurs from the P-ring to the I-ring. Consequently, a P-twisted intermediate will have (i) a more negative I-ring and (ii) a more positive P-ring than its pretwisted counterpart. Thus, it is expected that the surrounding solvent would rearrange to compensate for this CT process by shifting increased solvent density from near the $\mathrm{O}_{\mathrm{P}}$ to near the $\mathrm{O}_{\mathrm{I}}$. Interestingly, the opposite trend is absent for the I-species. We attribute this difference to the disparate deactivation time scales along $\phi_{I}$ and $\phi_{P}$ that are presented in Figure 9. Because deactivation through the I-channel occurs so rapidly, water does not have time to relax fully around I-twisted structures before internal conversion. Such behavior explains the absence of large shifts of excited-state RDF peaks in Figure 11a. On the other hand, deactivation through the P-channel is more gradual than decay through the I-channel, due to the barrier to reach the P-twisted CI seam from the corresponding TICT intermediate. Consequently, the solvent has time to reorient itself around P-twisted structures on the excited state. As a result, we observe gradual shifts of excited-state RDF peaks in Figure $11 \mathrm{~b}$.
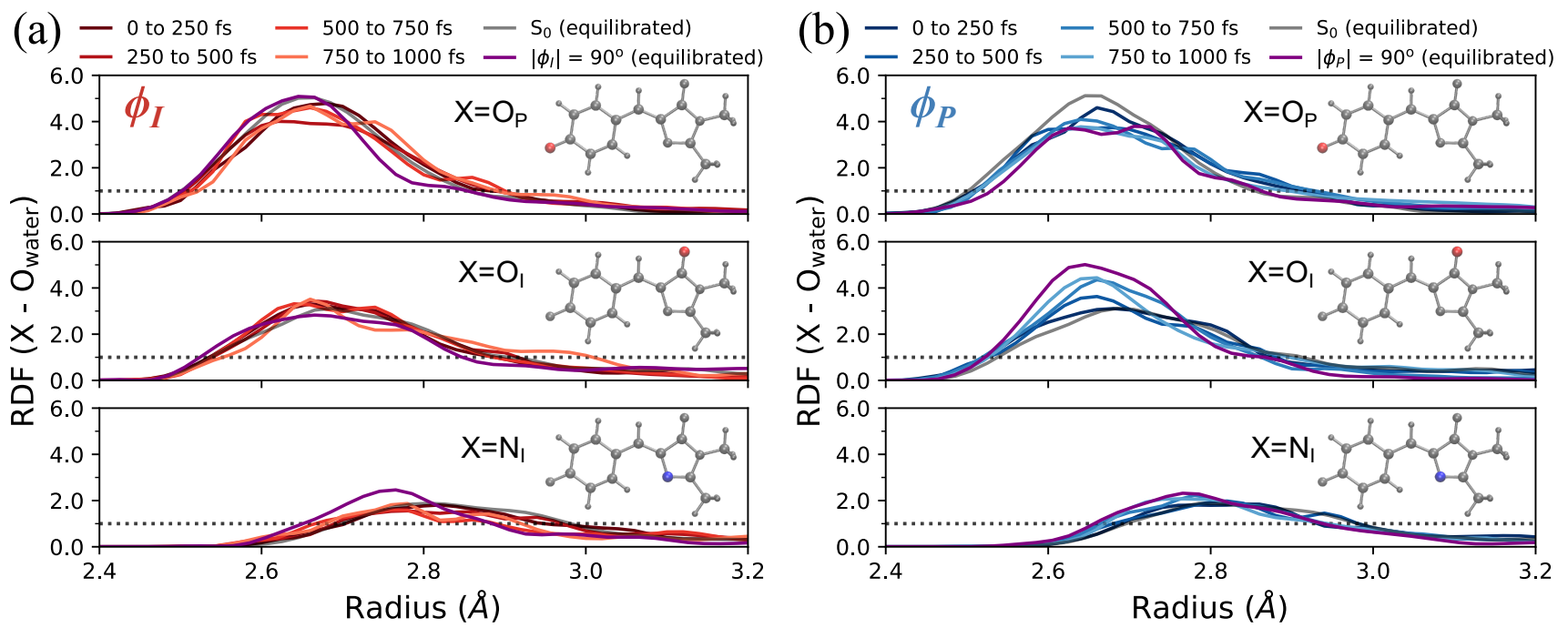

Figure 11: Radial distribution function (RDF) for HBDI- heteroatoms with water oxygen atoms during the first 1 ps of AIMS dynamics on $\mathrm{S}_{1}$. Each RDF shown for (a) I-deactivating species and (b) P-deactivating species begins and ends within the specified time window. Each figure focuses on the first solvation shell of the specified atom. The $\phi_{I}$-and $\phi_{P}$-twisted RDFs are obtained from umbrella sampling windows on $S_{1}$, with the specified angle restrained to $\pm 90^{\circ}$ while the other dihedral is restrained to zero degrees. The purple line represents the average of the $90^{\circ}$ - and $-90^{\circ}$-twisted RDFs on $\mathrm{S}_{1}$. 
A geometric analysis at the points of population transfer elucidates the difference between the deactivation profiles of the I- and P-species. For I-deactivation, the spawning events that lead to population transfer generally occur near geometries with $\phi_{I}$ angles near $90^{\circ}$ and $\phi_{P}$ angles that lie relatively close to planarity, and vice versa for P-deactivation (Figure 12). Noting the occurrence of spawning events in relation to the key dihedral and pyramidalization angles reveals that, in addition to lasting longer on $\mathrm{S}_{1}$, P-twisted TBFs demand larger pyramidalization angles than I-twisted TBFs to reach their respective CIs. To investigate the implications of this difference, the absolute population transfer was calculated for each spawning event as a function of the pyramidalization and dihedral angle values upon entering the spawning region (Figure 12a). Coupling these observations with AIMS data supports our claim that TBFs that reach highly I-twisted structures rapidly approach electronic degeneracy and transfer their $S_{1}$ population to the ground state. In contrast, the TBFs that proceed through P-twisting transfer their population less effectively and therefore persist on $\mathrm{S}_{1}$ for several picoseconds. Movie S1 provides a time lapse of these population and angle parameters to clarify this point. For a direct comparison of the spawned geometries associated with each pathway, the pyramidalization angle was measured for each spawned geometry of I-deactivating (Figure 12b) and Pdeactivating (Figure 12c) TBFs. From these distributions, it is clear that pyramidalization requirements for P-deactivating TBFs are more demanding (peaked at $\sim 30^{\circ}$ ) than the requirements for I-deactivating TBFs (peaked at $\sim 10^{\circ}$ ). The larger pyramidalization angles needed to reach the P-twisted CI gate access to the P-twisted region of the CI seam. We conclude that limited access to an uphill CI seam is responsible for the lingering $\mathrm{S}_{1}$ population of the P-species.

In addition to being more accessible than the P-twisted CI seam, the I-twisted CI seam appears to be more efficient at transferring population to the ground state. On average, population transfer events mediated by the I- and P-channels transfer $56 \pm 32 \%$ and $35 \pm 26 \%$ of the incoming $\mathrm{S}_{1}$ population to $\mathrm{S}_{0}$ per spawning event, respectively (Figure S12). This is expected based on the more direct approach toward the I-twisted CI seam, than for its P-twisted counterpart, as supported by Figures 5 and 6 . The uphill pyramidalization requirements and lack of efficiency explain the more gradual deactivation of the P-twisted species, when compared to the I-twisted species.

Relative to gas-phase $\mathrm{HBDI}^{-},{ }^{24}$ aqueous $\mathrm{HBDI}^{-}$generally demands less pyramidalization to reach I- and P-twisted CI seams (Figure 12b-c). This difference between systems becomes most apparent when comparing internal conversion tendencies associated with the I-channel. In gas-phase, I-twisted 
configurations transfer population to $\mathrm{S}_{0}$ while sampling along a larger range of $\phi_{P}$ values than in water. Based on our analysis regarding pyramidalization gating, we conclude that this behavior is due to the topology differences of the I-twisted CI seam across systems. Because solvation reduces the $\mathrm{S}_{0} / \mathrm{S}_{1}$ energy gap for I-twisted structures, aqueous $\mathrm{HBDI}^{-}$has smaller pyramidalization requirements to reach the I-twisted CI seam than does gas-phase HBDI-. Thus, the solvated chromophore spends little time sampling $\phi_{P}$ as it undergoes internal conversion through a near-barrierless process. The I-twisted spawning geometries of aqueous $\mathrm{HBDI}^{-}$, which are associated with ultrafast spawning tendencies, are consistent with rapid accessibility out of the FC region via an aborted hula-twist mechanism, as presented in Figure 8. Due to the larger pyramidalization angles required to reach the I-twisted CI seam in gas-phase, more extensive sampling along $\phi_{P}$ occurs for gas-phase HBDI- before the entire $\mathrm{S}_{1}$ population is transferred to $\mathrm{S}_{0}$. This logic extends to the comparison between I- and P-twisted spawning occurrences. Because reaching the P-twisted CI seam from a P-twisted TICT intermediate is an energetically uphill process, P-twisted intermediates significantly sample configurations above $90^{\circ}$. The enhanced sampling along this coordinate may explain the higher proportion of spawning geometries skewed toward the $E$-isomer, relative to this proportion for $\phi_{I}$ from I-twisted spawns (Figure S13).

(a)

a)

$S_{1}$ population: 1.00

$S_{1}$ population: 0.50

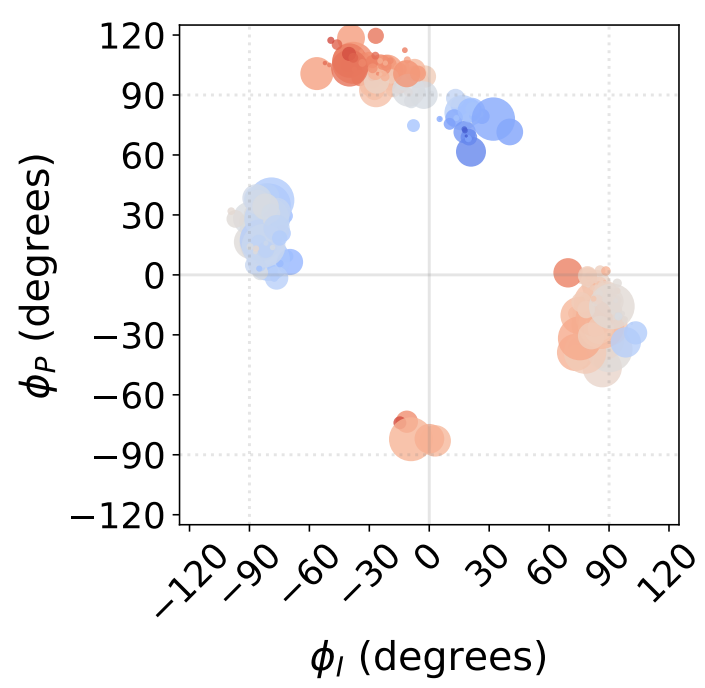

S population: 0.75

$S_{1}$ population: 0.25

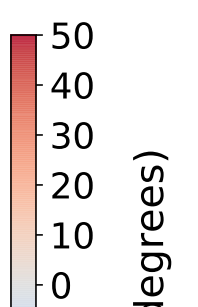

(c)

(c)

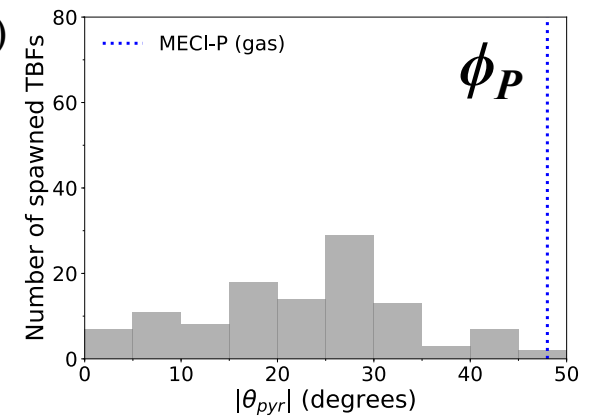

Figure 12: (a) Absolute population transfer and pyramidalization angle associated with the bridge carbon atom for spawned geometries of aqueous $\mathrm{HBDI}^{-}$from parent TBFs. Absolute population transfer is defined by the fraction of original $\mathrm{S}_{1}$ population transferred to $\mathrm{S}_{0}$. Plotted values of $\phi_{\mathrm{I}}, \phi_{\mathrm{P}}$, and $\theta_{\mathrm{pyr}}$ characterize geometries that initiate population transfer. A histogram (using $5^{\circ}$ bins) of pyramidalization angle magnitudes is provided for spawned TBFs that transfer population through torsion dominated by (b) $\phi_{\mathrm{I}}$ or (c) $\phi_{\mathrm{P}}$ dihedrals. Pyramidalization angles measured for I- and P-twisted MECIs in gasphase $^{24}$ are represented with vertical lines in (b) and (c). 
Based on vibrational signatures obtained from FSRS experiments, Taylor et al. proposed that the formation of a CS species and the transition from this CS state to a TICT state were responsible for measured short ( $\sim 350 \mathrm{fs})$ and long ( $\sim 2-3 \mathrm{ps})$ time components, respectively. ${ }^{31}$ Our data suggests a different origin of these time constants. As supported by the substantial torsional displacements sampled within the first $\sim 200$ fs (Figure 10), the formation of TICT states is essentially barrierless. In other words, we do not see evidence of a non-twisted CS intermediate in water. Rather, we observe two competing processes: (i) an I-twisting process that easily reaches a downhill CI seam and (ii) a Ptwisting process that encounters a barrier as it approaches an uphill CI seam. As an alternative, we propose that the different accessibility to the CI seams, from the corresponding TICT states associated with competing I- and P-twisted processes, is responsible for the short (caused by mainly I-species) and long (caused by mainly P-species) time components reported from the FSRS experiments. However, to rigorously establish this interpretation of the FSRS fingerprints requires calculation of the vibrational signatures of I- and P-twisted structures and remain a task for future work.

Photoproducts. To evaluate the branching ratio of HBDI- upon deactivation to the ground state, dynamics on $\mathrm{S}_{0}$ were continued for an additional $500 \mathrm{fs}$ (Figure S14, Table S5). Classifying the resulting products provides an estimate for the branching ratio with respect to $Z / E$ isomerization, $Z / Z$ isomerization, and ground-state recovery to the $Z$-isomer. Most of the population (87\%) returns to the $Z$ isomer (Figure 13), in line with the low $Z / E$ isomerization quantum yield reported by Vengris, et al. ${ }^{43}$ Despite I-torsion being the dominant deactivation pathway, associated CIs are highly inefficient at producing $E$ photoproducts. Thus, $\mathrm{HBDI}^{-}$is much less efficient at generating $E$ photoproducts in solution $\left(\sim 13 \%\right.$ yield) than in gas-phase $\left(\sim 40 \%\right.$ yield). ${ }^{24}$ Only $20 \%$ of the I-twisted spawns undergo $Z / E$ isomerization. P-twisted CIs are more efficient (37\%) at producing P-flipped products; however, the symmetry of the P-ring regenerates the $Z$-isomer whether or not the P-ring isomerizes. Verifying the $Z / Z$ isomerization quantum yield experimentally would require asymmetric substitution on the P-ring. The respective isomerization quantum yields are likely linked to the barrier (or lack thereof) between the TICT state and CI seam associated with each pathway. Because the I-twisted CI seam is reached without significant pyramidalization, I-twisted structures readily proceed through the CI seam as soon as twisting along $\phi_{I}$ nears $90^{\circ}$ (Figure S13a). On the other hand, the P-twisted CI seam is not reached so easily. As a consequence, P-twisted intermediates sample along $\phi_{P}$ more robustly to reach significantly pyramidalized structures. This results in a majority of spawns with $\phi_{P}$ greater than $90^{\circ}$ (Figure S13b) and likely contributes to the higher quantum yield for $Z / Z$ isomerization than that of $E / Z$ isomerization. 

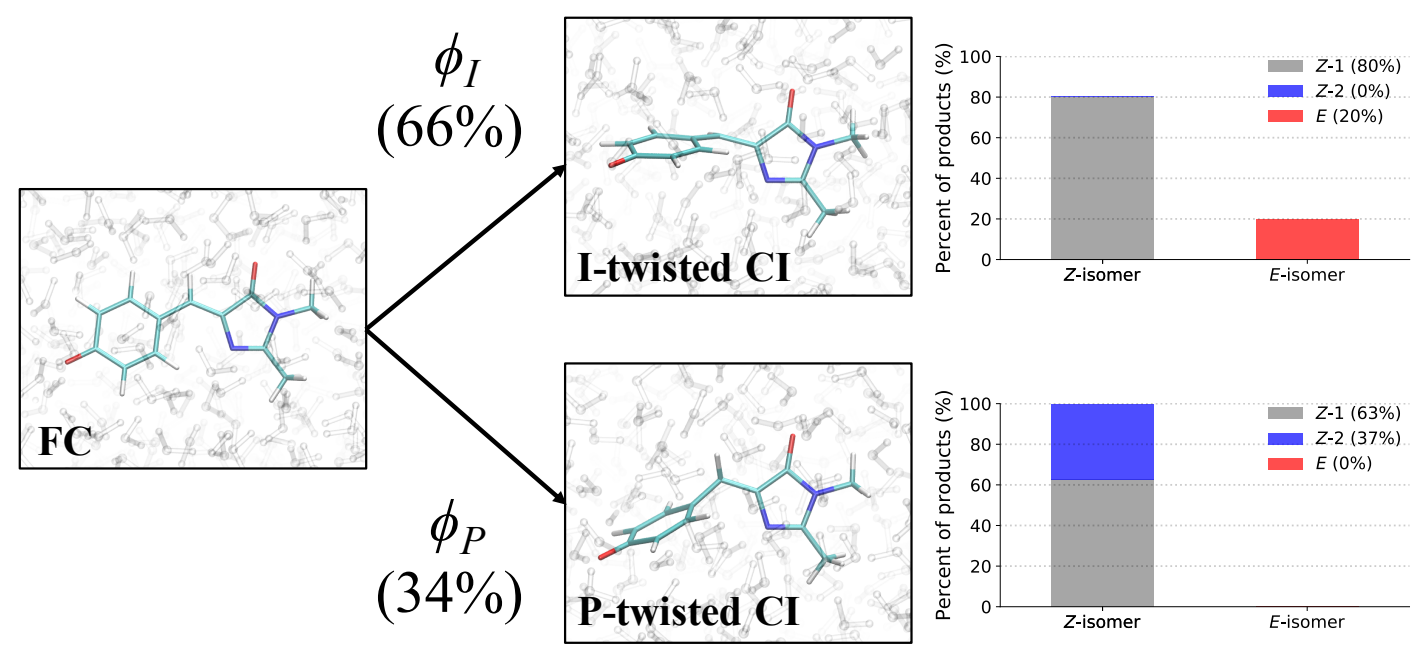

Figure 13: Photoproducts of $\mathrm{HBDI}^{-}$in water for spawned TBFs, following $500 \mathrm{fs}$ of QM/MM-MD on $\mathrm{S}_{0}$. A total of 264 spawned TBFs were analyzed. $\phi_{I}$ and $\phi_{P}$ branching percentages represent the fraction of TBFs that participated in each twisting pathway. Bar graphs illustrate the percentage of each product formed by the spawned TBFs for their respective pathway. The $Z-1$ product represents a $Z$-isomer of $\mathrm{HBDI}^{-}$that is not the result of $Z / Z$ isomerization, whereas the $Z-2$ product corresponds to a $Z$-isomer of $\mathrm{HBDI}^{-}$following $Z / Z$ isomerization.

Time-resolved fluorescence. Solvated $\mathrm{HBDI}^{-}$has been the subject of many time-resolved fluorescence experiments. ${ }^{27,37,102-104}$ To link our simulation data to experimental findings, time-resolved fluorescence spectra were computed over the course of the AIMS simulations (Figure 14). A line out at $500 \mathrm{~nm}$ (based on experiment ${ }^{99}$ ) is extracted for various sets of TBFs. Across all TBFs (Figure 14a), the two-dimensional fluorescence spectrum experiences a significant red-shift spectral evolution after a few hundred femtoseconds. The red-shift and the reduction in fluorescence signal are attributed to a decrease in the $\mathrm{S}_{0} / \mathrm{S}_{1}$ energy gap and emission strength, respectively, as the chromophore begins twisting its bridge dihedrals upon departure from the FC region (Table S3, Figure S16). Since twisting along $\phi_{I}$ and $\phi_{P}$ is correlated with a decrease in oscillator strength, we may expect the steeper (compared to XMSPT2) $\alpha$-CASSCF gradients beyond $30^{\circ}$ torsion about either the I or P bonds (Figure S1) to artificially accelerate fluorescence quenching in our AIMS simulations. The fluorescence spectrum was decomposed to obtain the contributions from the I- and P-deactivating species (Figure 14b, Figure 14c). By comparing the two-dimensional fluorescence spectra, it becomes obvious that the P-twisted channel significantly prolongs the fluorescence intensity of aqueous HBDI- when compared to the signal from the I-twisted channel. From the time-resolved fluorescence spectrum for all TBFs at $500 \mathrm{~nm}$ (Figure 14d), AIMS simulations predict a fluorescence signal that loses roughly $50 \%$ of its intensity by 500 fs. Despite this rapid quenching, fluorescence signatures are observed beyond 1.5 ps. An I- and P-pathway decomposition of this signal shows that I-twisting species quench their fluorescence rapidly (within a 
picosecond). Yet, the P-twisting species has a more pronounced fluorescence signal that survives until $\sim 1.6$ ps. Therefore, the long-lived signal in the time-resolved spectrum, across all TBFs, is attributed to the species that undergoes internal conversion through the P-channel. We attribute the faster fluorescence decay of our simulations, relative to experiment, to the slightly steeper $\alpha$-CASSCF gradients for partially twisted geometries, when compared to XMS-CASPT2. The underestimated $\mathrm{S}_{0} / \mathrm{S}_{1}$ energy gap for I- and P-twisted structures at the $\alpha$-CASSCF level (Figure S1) likely red-shifts the fluorescence signal of I- and P-twisted structures more than expected. However, this issue likely affects the fluorescence signal of P-twisted ICs, which remain on $\mathrm{S}_{1}$ for several picoseconds, more than the signal of I-twisted ICs. Another possible contributor to the faster-than-expected simulated fluorescence signal is a higher ratio of P-to-I species in experiment than sampled for our AIMS simulations, which would increase the intensity of the fluorescence tail. Despite these discrepancies, our simulations provide evidence that the deactivation pathway taken by the chromophore has a significant impact on the observed time-resolved fluorescence.

(a)

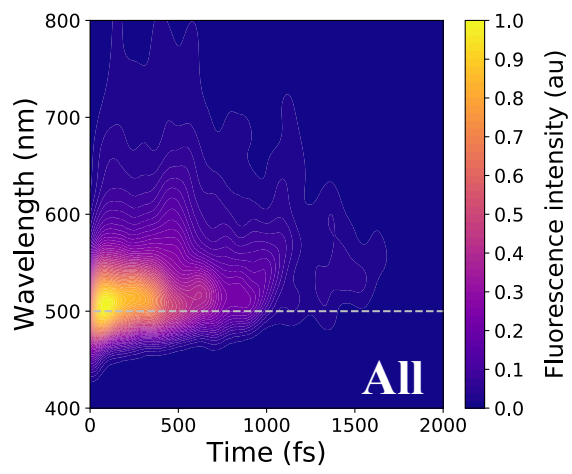

(c)

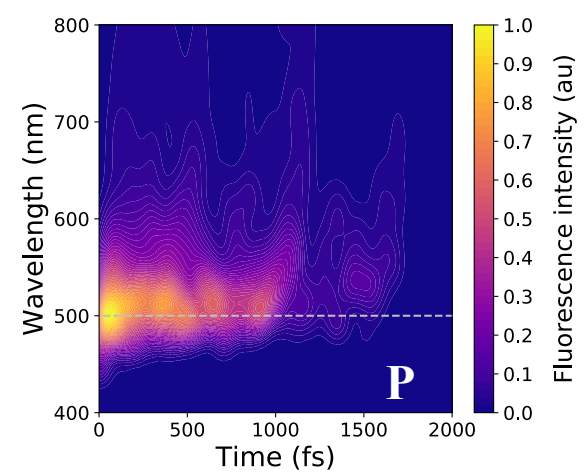

(b)

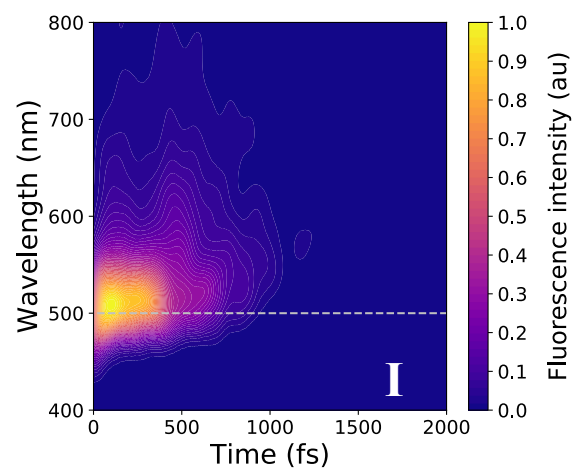

(d)

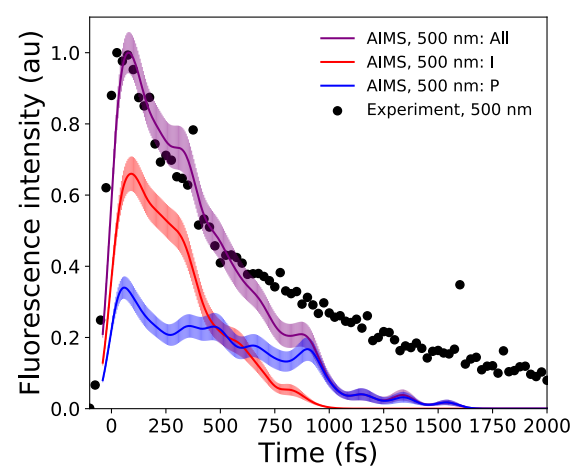

Figure 14: Two-dimensional fluorescence spectrum, probed at $500 \mathrm{~nm}$, for (a) all TBFs, (b) I-deactivating TBFs, and (c) Pdeactivating TBFs. (d) Time-resolved fluorescence signals of $\mathrm{HBDI}^{-}$calculated from AIMS simulations in water. The maximum fluorescence intensities of I- and P-deactivating species are scaled based on the relative proportion of ICs that deactivate through the I- and P-channels. Experimental data, following $400 \mathrm{~nm}$ excitation, for HBDI- in water was provided by Meech and co-workers. ${ }^{27,99}$ To align the experimental ${ }^{30}$ and theoretical fluorescence wavelength maxima, a shift of 0.13 $\mathrm{eV}$ was applied to the theory spectra (Figure S15). Transparent lines represent the error obtained from bootstrapping analysis. 


\section{Conclusions}

AIMS dynamics support the presence of two ground-state recovery mechanisms after the photoexcitation of the aqueous $\mathrm{HBDI}^{-}$to $\mathrm{S}_{1}$. The dominant mode of deactivation, through I-twisting, proceeds via an ultrafast mechanism and is responsible for the rapidly-decaying signals in time-resolved fluorescence and FSRS experiments. The minor pathway, through P-twisting, remains on $\mathrm{S}_{1}$ for several picoseconds and its access to the CI seam is gated by pyramidalization of the central methine bridge carbon. Our results provide evidence for the following mechanism: 1) evolution from the FC region via near-barrierless disrotatory motion (along $\phi_{I}$ and $\phi_{P}$ ) that is consistent with ground-state QM/MM-MD simulations, 2) formation of a TICT state through an aborted hula-twist mechanism as either $\phi_{I}$ or $\phi_{P}$ approaches $\left.90^{\circ}, 3\right)$ population transfer from $S_{1}$ to $S_{0}$ as the two states reach electronic degeneracy (through I-twisting for the I-species and through P-twisting along with pyramidalization for the Pspecies), and 4) re-population of the ground state with low $Z / E$ and $Z / Z$ isomerization quantum yields. This process is summarized in Figure 15. The ultrafast differentiation between I- and P-species indicates that both twisted species are accessible from the FC region with little/no energetic barrier, as supported by Figure 5 and Figure S1. Relative to the uphill approach needed to reach the P-twisted CI seam, the downhill approach to the I-twisted CI seam makes it easier to transfer population to the ground state through the I-channel. However, the P-twisted species has an isomerization quantum yield about two times as large as its I-twisted counterpart.

Our AIMS simulations provide novel mechanistic and electronic insight into the behavior that governs the photodynamics of the GFP chromophore. QM/MM-MD simulations indicate that surrounding $\mathrm{HBDI}^{-}$with mobile solvent molecules has minimal impact on the configuration space sampled by the chromophore in water. Yet, the electronic effects of water blue-shift the absorption spectrum. During gas- and solution-phase dynamics on $\mathrm{S}_{0}$, preferential twisting along $\phi_{\mathrm{I}}$ and $\phi_{\mathrm{P}}$ in a disrotatory fashion is observed. The subsequent internal conversion mechanisms predominantly occur through rotation of $\phi_{\mathrm{I}}$ in both systems. ${ }^{24}$ The absence of rigid electronic and steric constraints on the chromophore likely explains the ultrafast deactivation tendencies reported by experiments for both systems. ${ }^{21,27,30-31}$ However, the stabilization of highly-twisted structures and the promotion of electronic degeneracy, especially with respect to $\phi_{\mathrm{I}}$ which leads to a downhill CI seam, are responsible for shorter lifetimes in water when compared to gas-phase. Furthermore, the fitted time constants for the $S_{1}$ population decay $(609 \pm 181 \mathrm{fs}$ and $3.1 \pm 1.2 \mathrm{ps})$ are associated with internal conversion through the Iand P-channels, respectively. Through a direct comparison of AIMS simulations and experimental 
fluorescence spectra, we provide mechanistic insight to explain the observed fluorescence profile of HBDI $^{-}$in water. ${ }^{27}$ We conclude that the short experimental time constant (210 fs) fluorescence of HBDI ${ }^{-}$ in water is attributed to departure from the FC region (of both I- and P-deactivating species) and that the longer experimental fluorescence time constant (1.1 ps) corresponds to the longer-lived, P-deactivating species.

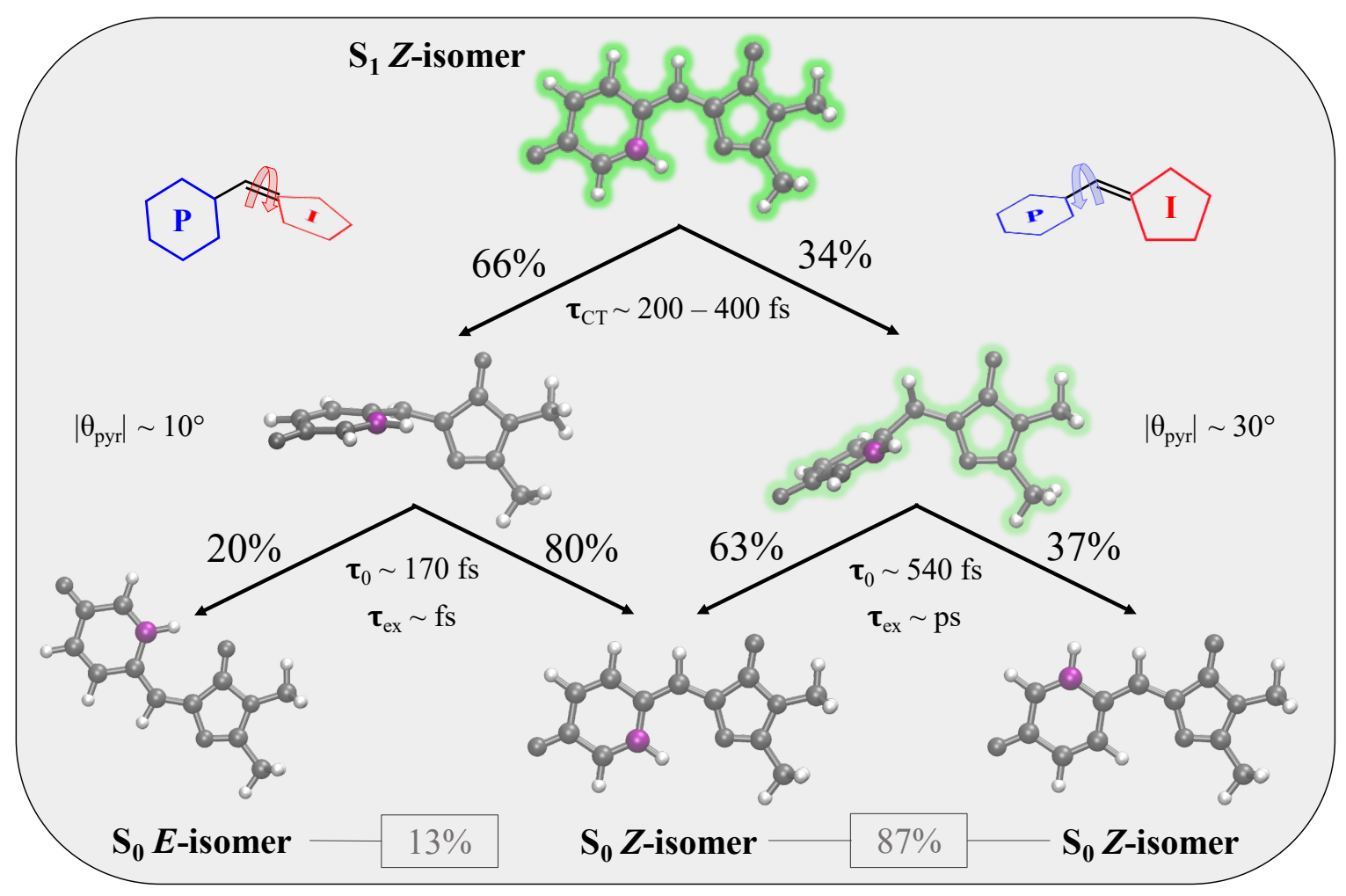

Figure 15: Summary of the photophysics of the solvated GFP chromophore. Percentages indicate the relative population that proceeds along a given pathway. $\boldsymbol{\tau}_{\mathrm{CT}}$ represents the time range at which TICT occurs, $\boldsymbol{\tau}_{0}$ represents the time at which population transfer begins, and $\boldsymbol{\tau}_{\mathrm{ex}}$ represents the order of the excited-state lifetime. $\theta_{\text {pyr }}$ represents the peak in the pyramidalization angle distribution for spawned geometries. Heavy atoms are colored gray, hydrogen atoms are colored white, and a reference carbon atom is colored purple to distinguish P-ring-flipped and original $Z$ products. Percentages listed in boxes indicate the total percent of spawned TBFs that formed the associated photoproduct.

Although our simulations do not include an explicit protein environment, our findings imply that the mobility and electronic distribution of residues surrounding the chromophore in a protein can tune the chromophore's behavior on the excited state. From our simulations, we predict that modifications to the protein environment can lead to internal conversion pathways with notably different population transfer rates, fluorescence profiles, and isomerization quantum yields. Using Dronpa2 variants, Romei and co-workers ${ }^{105}$ have demonstrated that chromophore modifications can bias the preferred 
directionality of the $\mathrm{CT}$ process and modify observables of interest in the protein. Our direct observation of TICT processes in solution provides evidence that steering the chromophore toward a given pathway can significantly alter its properties. To characterize the photochemical processes that drive the dynamics of the chromophore in protein scaffolds, we will extend our simulations and analyses to fluorescent protein systems.

\section{Author Contributions}

CMJ contributed to data curation, formal analysis, investigation, methodology, visualization and writing (original draft) of the presented work. NHL contributed to data curation, investigation, methodology and project administration. TJM contributed to methodology, project administration, funding acquisition, resources, and supervision. All authors contributed to conceptualization of the project and review and editing of the manuscript.

\section{Conflicts of Interest}

There are no conflicts to declare.

\section{Acknowledgements}

This work was supported by the AMOS program of the U.S. Department of Energy, Office of Science, Basic Energy Sciences, Chemical Sciences, and Biosciences Division. C.M.J. acknowledges financial support from the National Science Foundation graduate research fellowship program, and N.H.L. acknowledges financial support from the Villum Foundation (Grant No. VKR023371). C.M.J. also acknowledges Sergey Laptenok and Stephen Meech for providing additional experimental data. The authors also acknowledge Matthew Romei, Chi-Yun Lin, and Steven Boxer for their insight and discussions.

Jones, List, Martínez - Photodynamics of solvated HBDI anion - Page 27 


\section{References}

1. Shimomura, O.; Johnson, F. H.; Saiga, Y., Extraction, Purification and Properties of Aequorin, a Bioluminescent Protein from the Luminous Hydromedusan, Aequorea. Journal of Cellular and Comparative Physiology 1962, 59, 223-239.

2. Johnson, F. H.; Shimomura, O.; Saiga, Y.; Gershman, L. C.; Reynolds, G. T.; Waters, J. R., Quantum efficiency of Cypridina luminescence, with a note on that of Aequorea. Journal of Cellular and Comparative Physiology 1962, 60, 85-103.

3. Morin, J. G.; Hastings, J. W., Energy transfer in a bioluminescent system. Journal of Cellular Physiology 1971, 77, 313-318.

4. Morise, H.; Shimomura, O.; Johnson, F. H.; Winant, J., Intermolecular energy transfer in the bioluminescent system of Aequorea. Biochemistry 1974, 13, 2656-2662.

5. Shimomura, O., Structure of the chromophore of Aequorea green fluorescent protein. FEBS Letters 1979, 104, 220-222.

6. Chalfie, M.; Tu, Y.; Euskirchen, G.; Ward, W.; Prasher, D., Green fluorescent protein as a marker for gene expression. Science. 1994, 263, 802-805.

7. Inouye, S.; Tsuji, F. I., Aequorea green fluorescent protein. FEBS Letters 1994, 341, 277-280.

8. Tsien, R. Y., The Green Fluorescent Protein. Annual Review of Biochemistry 1998, 67, 509-544.

9. Feilmeier, B. J.; Iseminger, G.; Schroeder, D.; Webber, H.; Phillips, G. J., Green Fluorescent Protein Functions as a Reporter for Protein Localization in Escherichia coli. Journal of Bacteriology 2000, 182, 4068-4076.

10. Lippincott-Schwartz, J.; Snapp, E.; Kenworthy, A., Studying protein dynamics in living cells. Nature Reviews Molecular Cell Biology 2001, 2, 444-456.

11. Lippincott-Schwartz, J., Development and Use of Fluorescent Protein Markers in Living Cells. Science. 2003, 300, 87-91.

12. Ward, W. W.; Prentice, H. J.; Roth, A. F.; Cody, C. W.; Reeves, S. C., Spectral Perturbations of the Aequorea Green-Fluorescent Protein. Photochemistry and Photobiology 1982, 35, 803-808.

13. Heim, R.; Prasher, D. C.; Tsien, R. Y., Wavelength mutations and posttranslational autoxidation of green fluorescent protein. Proc. Natl. Acad. Sci. USA 1994, 91, 12501-12504.

14. Heim, R.; Cubitt, A. B.; Tsien, R. Y., Improved green fluorescence. Nature. 1995, 373, 663-664. 15. Heim, R.; Tsien, R. Y., Engineering green fluorescent protein for improved brightness, longer wavelengths and fluorescence resonance energy transfer. Current Biology 1996, 6, 178-182.

16. Dickson, R. M.; Cubitt, A. B.; Tsien, R. Y.; Moerner, W. E., On/off blinking and switching behaviour of single molecules of green fluorescent protein. Nature. 1997, 388, 355-358.

17. Patterson, G. H., A Photoactivatable GFP for Selective Photolabeling of Proteins and Cells. Science. 2002, 297, 1873-1877.

18. Hofmann, M.; Eggeling, C.; Jakobs, S.; Hell, S. W., Breaking the diffraction barrier in fluorescence microscopy at low light intensities by using reversibly photoswitchable proteins. Proceedings of the National Academy of Sciences 2005, 102, 17565-17569.

19. Kojima, S.; Ohkawa, H.; Hirano, T.; Maki, S.; Niwa, H.; Ohashi, M.; Inouye, S.; Tsuji, F. I., Fluorescent properties of model chromophores of tyrosine-66 substituted mutants of Aequorea green fluorescent protein (GFP). Tetrahedron Letters 1998, 39, 5239-5242.

20. Chattoraj, M.; King, B. A.; Bublitz, G. U.; Boxer, S. G., Ultra-fast excited state dynamics in green fluorescent protein: multiple states and proton transfer. Proceedings of the National Academy of Sciences 1996, 93, 8362-8367. 
21. Svendsen, A.; Kiefer, H. V.; Pedersen, H. B.; Bochenkova, A. V.; Andersen, L. H., Origin of the Intrinsic Fluorescence of the Green Fluorescent Protein. Journal of the American Chemical Society 2017, 139, 8766-8771.

22. Yang, F.; Moss, L. G.; Phillips, G. N., The molecular structure of green fluorescent protein. Nature Biotechnology 1996, 14, 1246-1251.

23. Ormö, M.; Cubitt, A. B.; Kallio, K.; Gross, L. A.; Tsien, R. Y.; Remington, S. J., Crystal Structure of the Aequorea victoria Green Fluorescent Protein. Science. 1996, 273, 1392-1395.

24. List, N. H.; Jones, C. M.; Martínez, T. J., Internal Conversion of the Anionic GFP Chromophore: In and Out of the I-twisted S1/S0 Conical Intersection Seam. doi: 10.26434/chemrxiv.14544555.

25. Perozzo, M. A.; Ward, K. B.; Thompson, R. B.; Ward, W. W., X-ray diffraction and timeresolved fluorescence analyses of Aequorea green fluorescent protein crystals. The Journal of biological chemistry 1988, 263, 7713-6.

26. Striker, G.; Subramaniam, V.; Seidel, C. A. M.; Volkmer, A., Photochromicity and Fluorescence Lifetimes of Green Fluorescent Protein. The Journal of Physical Chemistry B 1999, 103, 8612-8617.

27. Mandal, D.; Tahara, T.; Meech, S. R., Excited-State Dynamics in the Green Fluorescent Protein Chromophore. The Journal of Physical Chemistry B 2004, 108, 1102-1108.

28. Usman, A.; Mohammed, O. F.; Nibbering, E. T. J.; Dong, J.; Solntsev, K. M.; Tolbert, L. M., Excited-State Structure Determination of the Green Fluorescent Protein Chromophore. Journal of the American Chemical Society 2005, 127, 11214-11215.

29. West, C. W.; Hudson, A. S.; Cobb, S. L.; Verlet, J. R. R., Communication: Autodetachment versus internal conversion from the S1 state of the isolated GFP chromophore anion. The Journal of Chemical Physics 2013, 139, 071104.

30. Mooney, C. R. S.; Horke, D. A.; Chatterley, A. S.; Simperler, A.; Fielding, H. H.; Verlet, J. R. R., Taking the green fluorescence out of the protein: dynamics of the isolated GFP chromophore anion. Chem. Sci. 2013, 4, 921-927.

31. Taylor, M. A.; Zhu, L.; Rozanov, N. D.; Stout, K. T.; Chen, C.; Fang, C., Delayed vibrational modulation of the solvated GFP chromophore into a conical intersection. Physical Chemistry Chemical Physics 2019, 21, 9728-9739.

32. Forbes, M. W.; Jockusch, R. A., Deactivation Pathways of an Isolated Green Fluorescent Protein Model Chromophore Studied by Electronic Action Spectroscopy. Journal of the American Chemical Society 2009, 131, 17038-17039.

33. Niwa, H.; Inouye, S.; Hirano, T.; Matsuno, T.; Kojima, S.; Kubota, M.; Ohashi, M.; Tsuji, F. I., Chemical nature of the light emitter of the Aequorea green fluorescent protein. Proceedings of the National Academy of Sciences 1996, 93, 13617-13622.

34. Martin, M. E.; Negri, F.; Olivucci, M., Origin, Nature, and Fate of the Fluorescent State of the Green Fluorescent Protein Chromophore at the CASPT2//CASSCF Resolution. Journal of the American Chemical Society 2004, 126, 5452-5464.

35. Olsen, S.; Lamothe, K.; Martínez, T. J., Protonic Gating of Excited-State Twisting and Charge Localization in GFP Chromophores: A Mechanistic Hypothesis for Reversible Photoswitching. Journal of the American Chemical Society 2010, 132, 1192-1193.

36. Webber, N. M.; Litvinenko, K. L.; Meech, S. R., Radiationless Relaxation in a Synthetic Analogue of the Green Fluorescent Protein Chromophore. J. Phys. Chem. B 2001, 105, 8036-8039.

37. Litvinenko, K. L.; Webber, N. M.; Meech, S. R., Ultrafast Excited State Relaxation of the Chromophore of the Green Fluorescent Protein. Bulletin of the Chemical Society of Japan 2002, 75, 1065-1070. 
38. Weber, W.; Helms, V.; McCammon, J. A.; Langhoff, P. W., Shedding light on the dark and weakly fluorescent states of green fluorescent proteins. Proceedings of the National Academy of Sciences 1999, 96, 6177-6182.

39. Liu, R. S. H.; Asato, A. E., The primary process of vision and the structure of bathorhodopsin: a mechanism for photoisomerization of polyenes. Proceedings of the National Academy of Sciences 1985, $82,259-263$.

40. Altoe, P.; Bernardi, F.; Garavelli, M.; Orlandi, G.; Negri, F., Solvent Effects on the Vibrational Activity and Photodynamics of the Green Fluorescent Protein Chromophore: A Quantum-Chemical Study. Journal of the American Chemical Society 2005, 127, 3952-3963.

41. Park, J. W.; Shiozaki, T., On-the-Fly CASPT2 Surface-Hopping Dynamics. Journal of Chemical Theory and Computation 2017, 13, 3676-3683.

42. Minezawa, N.; Nakajima, T., Quantum mechanical/molecular mechanical trajectory surface hopping molecular dynamics simulation by spin-flip time-dependent density functional theory. The Journal of Chemical Physics 2020, 152, 024119.

43. Vengris, M.; van Stokkum, I. H. M.; He, X.; Bell, A. F.; Tonge, P. J.; van Grondelle, R.; Larsen, D. S., Ultrafast Excited and Ground-State Dynamics of the Green Fluorescent Protein Chromophore in Solution. The Journal of Physical Chemistry A 2004, 108, 4587-4598.

44. Levine, B. G.; Ko, C.; Quenneville, J.; Martinez, T. J., Conical intersections and double excitations in time-dependent density functional theory. Mol. Phys. 2006, 104, 1039-1051.

45. Kjonstad, E. F.; Myhre, R. H.; Martinez, T. J.; Koch, H., Crossing conditions in coupled cluster theory. J. Chem. Phys. 2017, 147, 164105.

46. Tuna, D.; Lefrancois, D.; Wolanski, L.; Gozem, S.; Schapiro, I.; Andruniow, T.; Dreuw, A.; Olivucci, M., Assessment of Approximate Coupled-Cluster and Algebraic-Diagrammatic-Construction Methods for Ground- and Excited-State Reaction Paths and the Conical-Intersection Seam of a RetinalChromophore Model. JCTC 2015, 11, 5758-5781.

47. Snyder, J. W.; Parrish, R. M.; Martínez, T. J., $\alpha$-CASSCF: An Efficient, Empirical Correction for SA-CASSCF To Closely Approximate MS-CASPT2 Potential Energy Surfaces. The Journal of Physical Chemistry Letters 2017, 8, 2432-2437.

48. Atchity, G. J.; Xantheas, S.; Ruedenberg, K., Potential energy surfaces near intersections. $J$. Chem. Phys. 1991, 95, 1862-1876.

49. Ben-Nun, M.; Molnar, F.; Schulten, K.; Martinez, T. J., The role of intersection topgraphy in bond selectivity of cis-trans photoisomerization. PNAS 2002, 99, 1769-1773.

50. Malhado, J. P.; Hynes, J. T., Non-adiabatic transition probability dependence on conical intersection topography. J. Chem. Phys. 2016, 145, 194104.

51. Virshup, A. M.; Chen, J.; Martinez, T. J., Nonlinear dimensionality reduction for nonadiabatic dynamics: The influence of conical intersection topography on population transfer rates. J. Chem. Phys. 2012, 137, 22A519.

52. Toniolo, A.; Granucci, G.; Martínez, T. J., Conical Intersections in Solution: A QM/MM Study Using Floating Occupation Semiempirical Configuration Interaction Wave Functions. The Journal of Physical Chemistry A 2003, 107, 3822-3830.

53. Toniolo, A.; Olsen, S.; Manohar, L.; Martínez, T. J., Conical intersection dynamics in solution: The chromophore of Green Fluorescent Protein. Faraday Discussions 2004, 127, 149-163.

54. Martinez, T. J.; Ben-Nun, M.; Levine, R. D., Multi-Electronic-State Molecular Dynamics: A Wave Function Approach with Applications. The Journal of Physical Chemistry 1996, 100, 7884-7895. 
55. Ben-Nun, M.; Martínez, T. J., Nonadiabatic molecular dynamics: Validation of the multiple spawning method for a multidimensional problem. The Journal of Chemical Physics 1998, 108, 72447257.

56. Ben-Nun, M.; Quenneville, J.; Martínez, T. J., Ab Initio Multiple Spawning: Photochemistry from First Principles Quantum Molecular Dynamics. The Journal of Physical Chemistry A 2000, 104, 5161-5175.

57. Ben-Nun, M.; Martínez, T. J., Ab Initio Quantum Molecular Dynamics. In Advances in Chemical Physics, 2002; Vol. 121, pp 439-512.

58. Curchod, B. F. E.; Martínez, T. J., Ab Initio Nonadiabatic Quantum Molecular Dynamics. Chemical Reviews 2018, 118, 3305-3336.

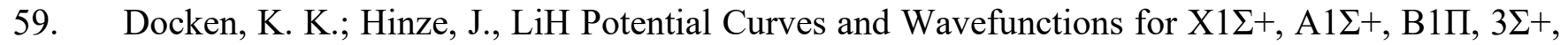
and 3П. The Journal of Chemical Physics 1972, 57, 4928-4936.

60. Roos, B. O., The Complete Active Space Self-Consistent Field Method and its Applications in Electronic Structure Calculations. In Adv. Chem. Phys., 2007; Vol. 69, pp 399-445.

61. Snyder, J. W.; Hohenstein, E. G.; Luehr, N.; Martínez, T. J., An atomic orbital-based formulation of analytical gradients and nonadiabatic coupling vector elements for the state-averaged complete active space self-consistent field method on graphical processing units. The Journal of Chemical Physics 2015, $143,154107$.

62. Snyder, J. W.; Fales, B. S.; Hohenstein, E. G.; Levine, B. G.; Martínez, T. J., A directcompatible formulation of the coupled perturbed complete active space self-consistent field equations on graphical processing units. The Journal of Chemical Physics 2017, 146, 174113.

63. Wolf, T. J. A.; Sanchez, D. M.; Yang, J.; Parrish, R. M.; Nunes, J. P. F.; Centurion, M.; Coffee, R.; Cryan, J. P.; Gühr, M.; Hegazy, K.; Kirrander, A.; Li, R. K.; Ruddock, J.; Shen, X.; Vecchione, T.; Weathersby, S. P.; Weber, P. M.; Wilkin, K.; Yong, H.; Zheng, Q.; Wang, X. J.; Minitti, M. P.; Martínez, T. J., The photochemical ring-opening of 1,3-cyclohexadiene imaged by ultrafast electron diffraction. Nature Chemistry 2019, 11, 504-509.

64. Frutos, L.; Andruniow, T.; Santoro, F.; Ferre, N.; Olivucci, M., Tracking the excited-state time evolution of the visual pigment with multiconfigurational quantum chemistry. PNAS 2007, 104, 7764.

65. Olsen, S.; McKenzie, R. H., A diabatic three-state representation of photoisomerization in the green fluorescent protein chromophore. The Journal of Chemical Physics 2009, 130, 184302.

66. Polyakov, I. V.; Grigorenko, B. L.; Epifanovsky, E. M.; Krylov, A. I.; Nemukhin, A. V., Potential Energy Landscape of the Electronic States of the GFP Chromophore in Different Protonation Forms: Electronic Transition Energies and Conical Intersections. Journal of Chemical Theory and Computation 2010, 6, 2377-2387.

67. Hehre, W. J.; Ditchfield, R.; Pople, J. A., Self-Consistent Molecular Orbital Methods. XII. Further Extensions of Gaussian-Type Basis Sets for Use in Molecular Orbital Studies of Organic Molecules. The Journal of Chemical Physics 1972, 56, 2257-2261.

68. Radhakrishnan, T. P.; Agranat, I., Measures of pyramidalization. Structural Chemistry 1991, 2, 107-115.

69. Finley, J.; Malmqvist, P.-Å.; Roos, B. O.; Serrano-Andrés, L., The multi-state CASPT2 method. Chemical Physics Letters 1998, 288, 299-306.

70. Granovsky, A. A., Extended multi-configuration quasi-degenerate perturbation theory: The new approach to multi-state multi-reference perturbation theory. The Journal of Chemical Physics 2011, 134, 214113. 
71. Song, C.; Martínez, T. J., Reduced scaling extended multi-state CASPT2 (XMS-CASPT2) using supporting subspaces and tensor hyper-contraction. The Journal of Chemical Physics 2020, 152, 234113.

72. Gidopoulos, N.; Theophilou, A., Hartree-Fock equations determining the optimum set of spin orbitals for the expansion of excited states. Philosophical Magazine B 1994, 69, 1067-1074.

73. Granucci, G.; Toniolo, A., Molecular gradients for semiempirical CI wavefunctions with floating occupation molecular orbitals. Chemical Physics Letters 2000, 325, 79-85.

74. Gidopoulos, N. I.; Papaconstantinou, P. G.; Gross, E. K. U., Ensemble-Hartree-Fock scheme for excited states. The optimized effective potential method. Physica B: Condensed Matter 2002, 318, 328332.

75. Slavíček, P.; Martínez, T. J., Ab initio floating occupation molecular orbital-complete active space configuration interaction: An efficient approximation to CASSCF. The Journal of Chemical Physics 2010, 132, 234102.

76. Kästner, J.; Carr, J. M.; Keal, T. W.; Thiel, W.; Wander, A.; Sherwood, P., DL-FIND: An OpenSource Geometry Optimizer for Atomistic Simulations. The Journal of Physical Chemistry A 2009, 113, 11856-11865.

77. Ufimtsev, I. S.; Martínez, T. J., Quantum Chemistry on Graphical Processing Units. 1. Strategies for Two-Electron Integral Evaluation. Journal of Chemical Theory and Computation 2008, 4, 222-231.

78. Ufimtsev, I. S.; Martinez, T. J., Quantum Chemistry on Graphical Processing Units. 2. Direct Self-Consistent-Field Implementation. Journal of Chemical Theory and Computation 2009, 5, 10041015 .

79. Ufimtsev, I. S.; Martinez, T. J., Quantum Chemistry on Graphical Processing Units. 3. Analytical Energy Gradients, Geometry Optimization, and First Principles Molecular Dynamics. Journal of Chemical Theory and Computation 2009, 5, 2619-2628.

80. Seritan, S.; Bannwarth, C.; Fales, B. S.; Hohenstein, E. G.; Kokkila-Schumacher, S. I. L.; Luehr, N.; Snyder, J. W.; Song, C.; Titov, A. V.; Ufimtsev, I. S.; Martínez, T. J., TeraChem: Accelerating electronic structure and ab initio molecular dynamics with graphical processing units. The Journal of Chemical Physics 2020, 152, 224110.

81. Seritan, S.; Bannwarth, C.; Fales, B. S.; Hohenstein, E. G.; Isborn, C. M.; Kokkila Schumacher, S. I. L.; Li, X.; Liu, F.; Luehr, N.; Snyder, J. W.; Song, C.; Titov, A. V.; Ufimtsev, I. S.; Wang, L.-P.; Martínez, T. J., TeraChem: A graphical processing unit - accelerated electronic structure package for large - scale ab initio molecular dynamics. WIREs Computational Molecular Science 2020, el494, 1-16.

82. Eastman, P.; Swails, J.; Chodera, J. D.; McGibbon, R. T.; Zhao, Y.; Beauchamp, K. A.; Wang, L.-P.; Simmonett, A. C.; Harrigan, M. P.; Stern, C. D.; Wiewiora, R. P.; Brooks, B. R.; Pande, V. S., OpenMM 7: Rapid development of high performance algorithms for molecular dynamics. PLOS Computational Biology 2017, 13, e1005659.

83. Wu, Y.; Tepper, H. L.; Voth, G. A., Flexible simple point-charge water model with improved liquid-state properties. The Journal of Chemical Physics 2006, 124, 024503.

84. Joung, I. S.; Cheatham III, T. E., Determination of Alkali and Halide Monovalent Ion Parameters for Use in Explicitly Solvated Biomolecular Simulations. J. Phys. Chem. B 2008, 112, 9020-9041.

85. Bayly, C. I.; Cieplak, P.; Cornell, W.; Kollman, P. A., A well-behaved electrostatic potential based method using charge restraints for deriving atomic charges: the RESP model. The Journal of Physical Chemistry 1993, 97, 10269-10280.

86. Lee, C.; Yang, W.; Parr, R. G., Development of the Colle-Salvetti correlation-energy formula into a functional of the electron density. Physical Review B 1988, 37, 785-789.

Jones, List, Martínez - Photodynamics of solvated HBDI anion - Page 32 
87. Becke, A. D., Density - functional thermochemistry. III. The role of exact exchange. The Journal of Chemical Physics 1993, 98, 5648-5652.

88. Grimme, S.; Antony, J.; Ehrlich, S.; Krieg, H., A consistent and accurate ab initio parametrization of density functional dispersion correction (DFT-D) for the 94 elements $\mathrm{H}-\mathrm{Pu}$. The Journal of Chemical Physics 2010, 132, 154104.

89. Wang, J.; Wolf, R. M.; Caldwell, J. W.; Kollman, P. A.; Case, D. A., Development and testing of a general amber force field. Journal of Computational Chemistry 2004, 25, 1157-1174.

90. Niklasson, A. M. N.; Steneteg, P.; Odell, A.; Bock, N.; Challacombe, M.; Tymczak, C. J.; Holmström, E.; Zheng, G.; Weber, V., Extended Lagrangian Born-Oppenheimer molecular dynamics with dissipation. The Journal of Chemical Physics 2009, 130, 214109.

91. Andersen, L. H.; Lapierre, A.; Nielsen, S. B.; Nielsen, I. B.; Pedersen, S. U.; Pedersen, U. V.; Tomita, S., Chromophores of the green fluorescent protein studied in the gas phase. The European Physical Journal D 2002, 20, 597-600.

92. Verlet, J. R. R., Private communication. 2019.

93. Efron, B.; Tibshirani, R. J., An Introduction to the Bootstrap. 1994.

94. McGibbon, R. T.; Beauchamp, K. A.; Harrigan, M. P.; Klein, C.; Swails, J. M.; Hernández, C. X.; Schwantes, C. R.; Wang, L.-p.; Lane, T. J.; Pande, V. S., MDTraj: A Modern Open Library for the Analysis of Molecular Dynamics Trajectories. Biophysical Journal 2015, 109, 1528-1532.

95. Roux, B., The calculation of the potential of mean force using computer simulations. Computer Physics Communications 1995, 91, 275-282.

96. Kumar, S.; Rosenberg, J. M.; Bouzida, D.; Swendsen, R. H.; Kollman, P. A., The weighted histogram analysis method for free-energy calculations on biomolecules. I. The method. Journal of Computational Chemistry 1992, 13, 1011-1021.

97. Grossfield, A. WHAM: an implementation of the weighted histogram analysis method. http://membrane.urmc.rochester.edu/wordpress/?page id=126 (accessed February 27, 2021).

98. Yu, J. K.; Liang, R.; Liu, F.; Martínez, T. J., First-Principles Characterization of the Elusive I Fluorescent State and the Structural Evolution of Retinal Protonated Schiff Base in Bacteriorhodopsin. Journal of the American Chemical Society 2019, 141, 18193-18203.

99. Laptenok, S.; Meech, S. R., Private communication. 2020.

100. Nielsen, S. B.; Lapierre, A.; Andersen, J. U.; Pedersen, U. V.; Tomita, S.; Andersen, L. H., Absorption Spectrum of the Green Fluorescent Protein Chromophore Anion In Vacuo. Physical Review Letters 2001, 87, 228102.

101. Jarzeba, W.; Walker, G. C.; Johnson, A. E.; Kahlow, M. A.; Barbara, P. F., Femtosecond Microscopic Solvation Dynamics of Aqueous-Solutions. Journal of Physical Chemistry 1988, 92 (25), 7039-7041.

102. Mandal, D.; Tahara, T.; Webber, N. M.; Meech, S. R., Ultrafast fluorescence of the chromophore of the green fluorescent protein in alcohol solutions. Chemical Physics Letters 2002, 358, 495-501.

103. Kummer, A. D.; Kompa, C.; Niwa, H.; Hirano, T.; Kojima, S.; Michel-Beyerle, M. E., ViscosityDependent Fluorescence Decay of the GFP Chromophore in Solution Due to Fast Internal Conversion. The Journal of Physical Chemistry B 2002, 106, 7554-7559.

104. Litvinenko, K. L.; Webber, N. M.; Meech, S. R., Internal Conversion in the Chromophore of the Green Fluorescent Protein: Temperature Dependence and Isoviscosity Analysis. The Journal of Physical Chemistry A 2003, 107, 2616-2623.

105. Romei, M. G.; Lin, C.-y.; Mathews, I. I.; Boxer, S. G., Electrostatic control of photoisomerization pathways in proteins. Science. 2020, 367, 76-79. 